\title{
Few-body resonances of unequal-mass systems with infinite interspecies two-body $s$-wave scattering length
}

\author{
D. Blume and K. M. Daily ${ }^{1}$ \\ ${ }^{1}$ Department of Physics and Astronomy, Washington State University, Pullman, Washington 99164-2814, USA
}

(Dated: November 13, 2018)

\begin{abstract}
Two-component Fermi and Bose gases with infinitely large interspecies $s$-wave scattering length $a_{s}$ exhibit a variety of intriguing properties. Among these are the scale invariance of two-component Fermi gases with equal masses, and the favorable scaling of Efimov features for two-component Bose gases and Bose-Fermi mixtures with unequal masses. This paper builds on our earlier work [D. Blume and K. M. Daily, arXiv:1006.5002] and presents a detailed discussion of our studies of small unequal-mass two-component systems with infinite $a_{s}$ in the regime where three-body Efimov physics is absent. We report on non-universal few-body resonances. Just like with twobody systems on resonance, few-body systems have a zero-energy bound state in free space and a diverging generalized scattering length. Our calculations are performed within a non-perturbative microscopic framework and investigate the energetics and structural properties of small unequalmass two-component systems as functions of the mass ratio $\kappa$, and the numbers $N_{1}$ and $N_{2}$ of heavy and light atoms. For purely attractive Gaussian two-body interactions, we find that the $\left(N_{1}, N_{2}\right)=(2,1)$ and $(3,1)$ systems exhibit three-body and four-body resonances at mass ratios $\kappa=12.314(2)$ and 10.4(2), respectively. The three- and four-particle systems on resonance are found to be large. This suggests that the corresponding wave function has relatively small overlap with deeply-bound dimers, trimers or larger clusters and that the three- and four-body systems on resonance have a comparatively long lifetime. Thus, it seems feasible that the features discussed in this paper can be probed experimentally with present-day technology.
\end{abstract}

PACS numbers: 03.75.Ss,05.30.Fk,34.50.-s

\section{INTRODUCTION}

Resonances arise in many different branches of physics. Near a resonance, certain physical observables are strongly enhanced, allowing one to probe the underlying physics at its "extreme". Resonances come in various types such as tunneling resonances (see, e.g., Ref. [1]), parametric resonances (see, e.g., Ref. [2]), stochastic resonances (see, e.g., Ref. [3]), or Fano-Feshbach resonances (see, e.g., Ref. [4]). Two-body Fano-Feshbach resonances play a key role in the study of ultracold atomic gases as they allow for the tuning of the interspecies and intraspecies $s$-wave scattering length $a_{s}$ essentially at will while leaving all other system parameters essentially unchanged $[5]$. In the ultracold regime, the scattering lengths determine the effective interaction strengths of the system. Thus, the tunability of $a_{s}$ allows for the realization of effectively repulsive and effectively attractive systems. Furthermore, the interaction strength can be tuned such that the system is effectively non-interacting or infinitely strongly interacting. Recent experiments that rely on this tunability include the study of the BCS-BEC crossover problem [9-15], the polaron problem [16], and the creation of bosonic and fermionic Feshbach molecules [12, 17-20]. These Feshbach molecules form an ideal starting point for creating polar molecules in the rovibrational ground state $21-23$.

Fano-Feshbach resonances have also played a key role in recent experiments aimed at studying few-body physics. The most prominent example is the observation of Efimov physics [24 31], which relies on tuning the $s$ - wave scattering length over several orders of magnitude. Efimov physics manifests itself most clearly in the vicinity of resonances. In particular, Efimov physics in the bosonic three-particle sector has been studied through the observation of enhanced three-body recombination loss rates near atom-dimer and atom-atom-atom resonances [27, 28]. An atom-dimer resonance exists when the binding energy of the Efimov trimer equals that of the dimer. An atom-atom-atom resonance exists when the Efimov trimer has a zero-energy binding energy, i.e., sits right at threshold. Similarly, in the bosonic four-particle sector, the expected scaling associated with Efimov physics has been confirmed through measurements of enhanced four-body recombination loss rates at atom-atom-atom-atom, atom-trimer and dimer-dimer resonances 29 33. An intriguing aspect of the features associated with Efimov physics is that the characterization of the three- and four-body sectors requires, for onecomponent Bose gases, just two parameters, the $s$-wave scattering length $a_{s}$ and a three-body parameter, which can for example be parametrized in terms of the binding energy of one of the Efimov trimers [32, 34]. These two parameters determine the positions of all universal resonances eluded to above. Efimov physics can also dominate the behavior of fermionic systems consisting of two or more components and of Bose-Fermi mixtures if the system parameters are tuned appropriately [35 41].

This paper investigates few-body resonances for twocomponent systems with infinitely large interspecies $s$ wave scattering length $a_{s}$ in the regime where three-body Efimov physics is absent. Building on earlier work [35- 
37, 42 49], we address the following questions: (Q1) Under which conditions do atom-atom-atom resonances occur for the $(2,1)$ system? (Q2) Under which conditions do atom-atom-atom-atom resonances occur for the $(3,1)$ system? (Q3) If the resonances discussed in Q1 and Q2 exist, what are their characteristics? (Q4) What are the differences and commonalities of system properties derived for finite-range (FR) and zero-range ( $\mathrm{ZR}$ ) interactions? (Q5) What are the implications of our theoretical studies for experiment [50]?

The remainder of this paper is organized as follows. Section $\llbracket \mathrm{A}$ introduces the system Hamiltonian while Secs. II B and II C discuss two complementary approaches for solving the time-independent Schrödinger equation for small two-component systems with unequal masses. Section [II presents our results for the energetics and selected structural properties for various parameter combinations. Lastly, Section IV summarizes our main results and concludes. A discussion of the main ideas and results of our work can be found in Ref. [48]. The present paper elaborates on the theoretical framework and provides a more detailed discussion of the results and their implications. In addition, the present paper presents structural properties, detailed comparisons between observables derived within the numerical and analytical frameworks, and results for the $(3,2)$ and $(4,1)$ systems.

\section{THEORETICAL BACKGROUND}

This section introduces and discusses the system Hamiltonian that underlies our studies, and the techniques employed to solve the time-independent Schrödinger equation associated with this Hamiltonian. Our solutions to the Schrödinger equation are obtained following two distinctly different approaches. On the one hand, we pursue an analytical treatment that employs hyperspherical coordinates and determines the solution in terms of some unknown. On the other hand, we solve the Schrödinger equation numerically. The numerical solutions are then used to determine some of the unknowns that arise in the first approach, thereby providing an interpretation of the numerical results and, conversely, a check of the analytical framework.

\section{A. System Hamiltonian}

We consider two-component systems with $N$ particles, where $N=N_{1}+N_{2}$, under external spherically symmetric harmonic confinement with angular trapping frequency $\omega$. If $N_{i}=1(i=1$ or 2$)$, no permutation symmetry of the $i$ th species needs to be imposed; this implies that the single particle species can be fermionic or bosonic. In contrast, if $N_{i}$ is greater than 1 , the results depend on the permutation symmetry. Throughout, we impose fermionic symmetry for systems with $N_{i}>1$.
The masses of the two species are denoted by $m_{1}$ and $m_{2}$, and our model Hamiltonian $H$ reads

$$
\begin{gathered}
H=\sum_{j=1}^{N_{1}}\left(\frac{-\hbar^{2}}{2 m_{1}} \nabla_{\vec{r}_{j}}^{2}+\frac{1}{2} m_{1} \omega^{2} \vec{r}_{j}^{2}\right)+ \\
\sum_{j=N_{1}+1}^{N}\left(\frac{-\hbar^{2}}{2 m_{2}} \nabla_{\vec{r}_{j}}^{2}+\frac{1}{2} m_{2} \omega^{2} \vec{r}_{j}^{2}\right)+\sum_{j=1}^{N_{1}} \sum_{k=N_{1}+1}^{N} V_{\mathrm{tb}}\left(r_{j k}\right) \cdot(1)
\end{gathered}
$$

Here, $\vec{r}_{j}$ denotes the position vector of the $j$ th particle measured with respect to the trap center and $V_{\mathrm{tb}}\left(r_{j k}\right)$ with $r_{j k}=\left|\vec{r}_{j}-\vec{r}_{k}\right|$ the interspecies interaction potential. Throughout, we assume that the particles of the same species are effectively non-interacting, i.e., we neglect intraspecies interactions. In the case of fermionic species this assumption is fullfilled with high accuracy for most systems since $s$-wave interactions are forbidden by symmetry and $p$-wave interactions are naturally suppressed [51].

Although nature provides us with only a finite number of discrete mass ratios $\kappa$, where

$$
\kappa=m_{1} / m_{2},
$$

we find it useful to treat $\kappa$ as a continuous variable to unravel the key physics, i.e., to see how the physics changes as a particularly interesting mass ratio is approached. Experimentally, the effective mass ratio of two-component systems could be tuned by loading the system into an optical lattice [44]. In addition to the mass ratio, we vary the number of heavy and light particles, the angular momentum and parity of the state under consideration, and the two-body interaction $V_{\mathrm{tb}}$.

Our calculations are performed for two classes of interaction potentials, FR and ZR interactions. Our FR calculations are performed for a purely attractive Gaussian potential $V_{\mathrm{g}}$ with range $r_{0}$ and depth $V_{0}\left(V_{0}>0\right)$,

$$
V_{\mathrm{g}}(r)=-V_{0} \exp \left[-\left(\frac{r}{\sqrt{2} r_{0}}\right)^{2}\right] \text {. }
$$

While the majority of our FR calculations considers the infinite scattering length limit, i.e., $1 / a_{s}=0$, we also explore how the system behavior changes as $\left|a_{s}\right|$ is decreased. In particular, we pick $r_{0}$ and then adjust the depth $V_{0}$ so that the two-body potential has the desired free-space $s$-wave scattering length $a_{s}$. For positive $a_{s}$, we restrict ourselves to potentials that support a single $s$-wave two-body bound state. For negative $a_{s}$, we restrict ourselves to potentials that support no two-body bound state. Our ZR calculations employ the FermiHuang pseudopotential $V_{\text {zr }}$ [52, 53],

$$
V_{\mathrm{zr}}(r)=\frac{2 \pi \hbar^{2} a_{s}}{\mu} \delta(\vec{r}) \frac{\partial}{\partial r} r,
$$

where $\mu$ denotes the reduced mass,

$$
\mu=m_{1} m_{2} /\left(m_{1}+m_{2}\right)
$$


Our ZR calculations are restricted to unitarity, i.e., to the regime where the $s$-wave scattering length is infinitely large and where the two-body system in free space is at the verge of supporting a zero-energy bound state.

The system under study is characterized by the following length scales: the harmonic oscillator length $a_{\text {ho }}$,

$$
a_{\mathrm{ho}}=\sqrt{\hbar /(2 \mu \omega)},
$$

the range $r_{0}$ of the interaction potential $\left(r_{0}=0\right.$ for $\left.V_{\mathrm{zr}}\right)$, and the $s$-wave scattering length $a_{s}$. At unitarity, the $s$-wave scattering length no longer defines a meaningful length scale. For sufficiently large $\kappa$, an additional length scale is given by the generalized $N$-body scattering length (see Sec. IIB). Our FR calculations are performed in the regime where $r_{0} \ll a_{\mathrm{ho}}$. In fact, one of the goals of this paper is to quantify how observables obtained for FR interactions approach those determined in the ZR limit. As will be shown in Sec.III, FR effects can be appreciable for two-component unequal-mass systems.

Our approaches outlined in Secs. IB and IIC take advantage of the fact that the center-of-mass degrees of freedom $\vec{R}_{\text {cm }}$ separate off. Throughout, we assume that the center-of-mass wave function is in the ground state, and we label the solutions $\Psi$ to the time-independent Schrödinger equation in the relative coordinates by the relative orbital angular momentum $L$ and the relative parity $\Pi$. The corresponding relative eigenenergies will be denoted by $E$. The Hamiltonian given in Eq. (1) describes the trapped system. As we discuss in Sec. [IB the eigenenergies of the trapped $N$-body system can be expressed in terms of the generalized scattering length that characterizes the corresponding $N$-body scattering problem in free space. This connection between the trapped and free-space systems, which is of course well known for the two-body problem [54], provides a great deal of insight.

\section{B. Hyperspherical coordinate treatment}

Within the hyperspherical framework [55 58], the $3 N-3$ relative coordinates are divided into $3 N-4$ hyperangles, collectively denoted by $\vec{\Omega}$, and a single length, the hyperradius $R, \mu R^{2}=\sum_{j=1}^{N} m_{j}\left(\vec{r}_{j}-\vec{R}_{\mathrm{cm}}\right)^{2}$. In the present context, these hyperspherical coordinates are particularly appealing since the relative wave function $\Psi$ of the unitary system with ZR interactions has been shown to separate for any number of particles into a hyperradial part $F_{\nu q}(R)$ and a hyperangular part $\Phi_{\nu}(\vec{\Omega})[42], \Psi_{\nu q}(R, \vec{\Omega})=R^{-(3 N-4) / 2} F_{\nu q}(R) \Phi_{\nu}(\vec{\Omega})$. Here, $\nu$ and $q$ denote hyperangular and hyperradial quantum numbers for a given angular momentum $L$ and parity $\Pi ; \nu$ takes the values $0,1, \cdots$ while $q$ takes non-integer values (see below). For ZR interactions with $1 / a_{s}=0$, the relative Schrödinger equation can therefore be solved in a two-step process. First, the hyperangular functions $\Phi_{\nu}(\vec{\Omega})$, or so-called channel functions, are determined

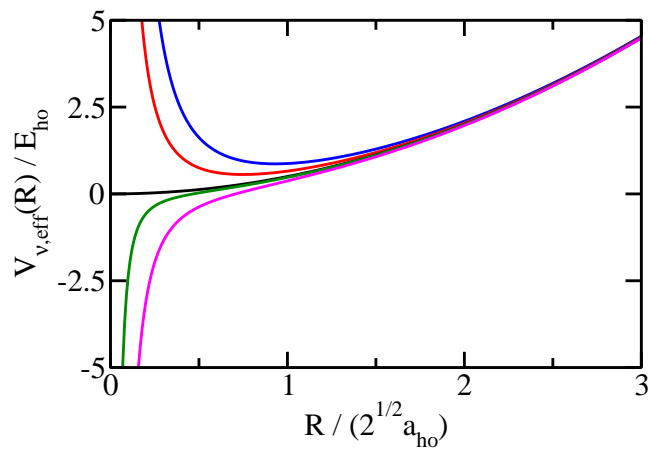

FIG. 1: (Color online) Effective hyperradial potential curve $V_{\nu, \text { eff }}(R)$, Eq. (7), as a function of $R$ for, from top to bottom, $s_{\nu}=1,3 / 4,1 / 2,9 / 20$ and 0 .

by solving the hyperangular Schrödinger equation [42]. The corresponding eigenvalues are related to the coefficients $s_{\nu}$, which determine the effective potential curves $V_{\nu, \mathrm{eff}}(R)$,

$$
V_{\nu, \mathrm{eff}}(R)=\frac{\hbar^{2}\left(s_{\nu}^{2}-1 / 4\right)}{2 \mu R^{2}}+\frac{1}{2} \mu \omega^{2} R^{2} .
$$

Figure 1 shows the effective potential curves $V_{\nu \text {,eff }}(R)$ for different $s_{\nu}$. For $s_{\nu}=1 / 2$, the hyperangular $1 / R^{2}$ term vanishes and $V_{\nu \text {,eff }}(R)$ reduces to the trapping potential, i.e., the second term on the right hand side of Eq. (77). For $s_{\nu}>1 / 2$ and $s_{\nu}<1 / 2$, the hyperangular $1 / R^{2}$ term is repulsive and attractive, respectively, and dominates at small $R$. Second, the hyperradial Schrödinger equation

$$
\left(\frac{-\hbar^{2}}{2 \mu} \frac{\partial^{2}}{\partial R^{2}}+V_{\nu, \mathrm{eff}}(R)\right) F_{\nu q}(R)=E_{\nu q} F_{\nu q}(R)
$$

is solved for $F_{\nu q}(R)$ and $E_{\nu q}$. If one uses FR instead of ZR interactions, the hyperangular and hyperradial parts of the wave function $\Psi$, in general, do not fully separate, implying non-vanishing coupling matrix elements between the different hyperangular channel functions. In a first approximation, however, these couplings can be neglected if $r_{0} \ll a_{\mathrm{ho}}$. The framework outlined in this section is thus not only applicable to ZR interaction but also, at least within an approximative scheme, to FR interactions (see Refs. [59, 60]).

Solving the hyperangular Schrödinger equation, i.e., determining the $s_{\nu}$, is, in general, a non-trivial task. For the three-body system with ZR interactions and infinitely large $a_{s}$, however, the eigenvalues can be obtained by solving a simple transcendental equation for each $L^{\Pi}$ symmetry [24, 25, 46, 47, 61]. Figure 2 shows the $s_{0}$ coefficients for the $(2,1)$ system, i.e., the system with two heavy fermions and one light atom, at unitarity with $L=0-3$ and $\Pi=(-1)^{L}$ as a function of the mass ratio $\kappa$. The state with the smallest $s_{0}$ value has $L^{\Pi}=1^{-}$ 


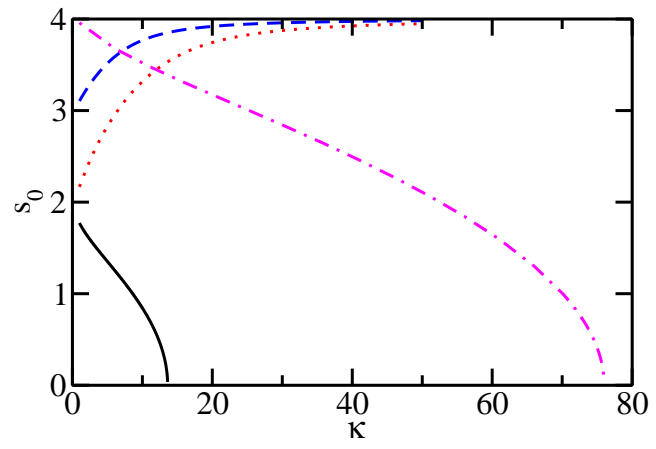

FIG. 2: (Color online) $s_{0}$ coefficient as a function of $\kappa$ for the $(2,1)$ system at unitarity with ZR interactions and $L^{\Pi}=0^{+}$ (dotted line), $L^{\Pi}=1^{-}$(solid line), $L^{\Pi}=2^{+}$(dashed line) and $L^{\Pi}=3^{-}$(dash-dotted line). Three-body Efimov physics is absent if $s_{0}>0$, which corresponds to $\kappa \lesssim 13.607$ for the $L^{\Pi}=1^{-}$system and to $\kappa \lesssim 76.0$ for the $L^{\Pi} \stackrel{\sim}{=} 3^{-}$system.

symmetry for mass ratios $\kappa \lesssim 13.607$ (solid line in Fig. (2). The coefficient $s_{0}$ decreases from 1.773 for $\kappa=1$ to 0 for $\kappa \approx 13.607$. For later reference, we note that $s_{0}$ equals 1 for $\kappa \approx 8.619$ and $1 / 2$ for $\kappa=12.313$. For $\kappa \gtrsim 13.607$, $s_{0}$ becomes purely imaginary and Efimov physics comes into play [24, 25, 35 37]. The $s_{0}$ coefficient of other odd $L$ states (the dash-dotted line shows the $s_{0}$ coefficient for the $L^{\Pi}=3^{-}$state) becomes imaginary for much larger mass ratios. For even $L$ states, $s_{0}$ increases with increasing $\kappa$ and approaches an integer value in the large $\kappa$ limit. For larger systems with infinitely large interspecies $s$-wave scattering length, the $s_{\nu}$ coefficients are not known in general.

We now consider the hyperradial Schrödinger equation, Eq. (8), for the $N$-body system, which can be solved for arbitrary $s_{\nu}$. Generalizing the quantum defect theory type approach from Ref. [62] from $s_{\nu}=1 / 2$ to arbitrary $s_{\nu}$, the solution $F_{\nu q}(R)$ to the second order differential equation can be written as

$$
F_{\nu q}(R)=N_{\nu q}\left[f_{\nu q}(R)-\tan \left(\pi \mu_{\nu q}\right) g_{\nu q}(R)\right],
$$

where $N_{\nu q}$ denotes a normalization constant. The quantum defect $\mu_{\nu q}$ determines the relative contributions of the regular solution $f_{\nu q}$ and the irregular solution $g_{\nu q}, f_{\nu q}(x)=A_{\nu q} x^{s_{\nu}+1 / 2} \exp \left(-x^{2} / 2\right)_{1} F_{1}\left(-q, s_{\nu}+\right.$ $\left.1, x^{2}\right)$ and $g_{\nu q}(x)=B_{\nu q} x^{-s_{\nu}+1 / 2} \exp \left(-x^{2} / 2\right)_{1} F_{1}(-q-$ $\left.s_{\nu},-s_{\nu}+1, x^{2}\right)$. Here, $A_{\nu q}$ and $B_{\nu q}$ denote constants [63], and $x$ the dimensionless hyperradial coordinate, $x=$ $R /\left(\sqrt{2} a_{\mathrm{ho}}\right)$. The non-integer quantum number $q$ is related to the eigenenergy $E_{\nu q}$ through

$$
E_{\nu q}=\left(2 q+s_{\nu}+1\right) \hbar \omega
$$

To determine the allowed values of $\mu_{\nu q}$, we enforce that $F_{\nu q}(x)$ vanishes at large $x$, resulting in the condition $\sin \left[\pi\left(\mu_{\nu q}+q\right)\right]=0$. This condition allows $\mu_{\nu q}$ to be eliminated, leaving the quantum number $q$ as the only unknown.

The quantization condition, i.e., the allowed $q$ values, are determined by investigating the small $x$ behavior of $F_{\nu q}(x)$. For $x \rightarrow 0, f_{\nu q}(x)$ behaves as $x^{s_{\nu}+1 / 2}$ and is well-behaved or less strongly diverging than $x^{-1 / 2}$ for $s_{\nu}>-1$ while $g_{\nu q}(x)$ behaves as $x^{-s_{\nu}+1 / 2}$ and diverges faster than $x^{-1 / 2}$ for $s_{\nu}>1$. Thus, we consider the regimes $s_{\nu}>1$ and $0<s_{\nu}<1$ separately (the Efimov regime with imaginary $s_{\nu}$ is not treated in this paper). For $s_{\nu}>1, g_{\nu q}(x)$ must be eliminated [64] and $F_{\nu q}(x)$ is determined by the exponentially decaying piece of $f_{\nu q}(x)$. The quantization condition becomes, in agreement with Ref. [42], $q=0,1, \cdots$ and the corresponding energy is referred to as $E_{f, \nu q}$,

$$
E_{f, \nu q}=\left(2 q+s_{\nu}+1\right) \hbar \omega ; q=0,1, \cdots .
$$

For $0<s_{\nu}<1$, both $f_{\nu q}(x)$ and $g_{\nu q}(x)$ are well-behaved and the allowed $q$ values depend on the boundary condition at small $x$. This boundary condition is determined by the true atom-atom interactions and cannot be derived within the ZR framework. Similarly to the case of Efimov trimers [24 26], the value of the short-range hyperradial boundary condition can be thought of as an extra parameter that is needed to specify the solution. In our case, this parameter characterizes the $N$-body system and can be parameterized, e.g., by the logarithmic derivative $L_{\nu q}\left(x_{0}\right), L_{\nu q}\left(x_{0}\right)=\left[\left(\partial F_{\nu q}(x) / \partial x\right) / F_{\nu q}(x)\right]_{x=x_{0}}$.

For $s_{\nu}>0\left(s_{\nu}\right.$ not equal to an integer $)$, the normalized hyperradial wave function $F_{\nu q}(R)$ can be compactly written as

$$
\begin{array}{r}
F_{\nu q}(x)= \\
N_{\nu q} \exp \left(\frac{-x^{2}}{2}\right) x^{s_{\nu}+1 / 2} U\left(-q, s_{\nu}+1, x^{2}\right),
\end{array}
$$

where $U$ denotes the confluent hypergeometric function of the second kind and

$$
N_{\nu q}=\sqrt{\frac{-2 \sin \left(\pi s_{\nu}\right) \Gamma(1-q) \Gamma\left(-q-s_{\nu}\right)}{\pi+\pi^{2} q \cot (\pi q)+q \pi\left[\psi(q)-\psi\left(-q-s_{\nu}\right)\right]}} ;
$$

here, $\psi$ denotes the digamma function 65]. Using Eq. (12), the logarithmic derivative at $x=x_{0}$ can be written as

$$
\begin{array}{r}
L_{\nu q}\left(x_{0}\right)=\frac{\frac{1}{2}-s_{\nu}}{x_{0}}- \\
x_{0}+\frac{2\left(q+s_{\nu}\right)}{x_{0}} \frac{U\left(-q, s_{\nu}, x_{0}^{2}\right)}{U\left(-q, s_{\nu}+1, x_{0}^{2}\right)} .
\end{array}
$$

Figure 3(a) shows the quantum number $q$ for $s_{\nu}=3 / 5$ for $x_{0} \ll 1$ (i.e., $x_{0}=1 \times 10^{-3}, 2 \times 10^{-3}$ and $3 \times 10^{-3}$ ) as a function of the inverse of the logarithmic derivative. $q$ is approximately equal to integer values $(q \approx 0,1, \cdots)$ for nearly all values of the logarithmic derivative. Exceptions 

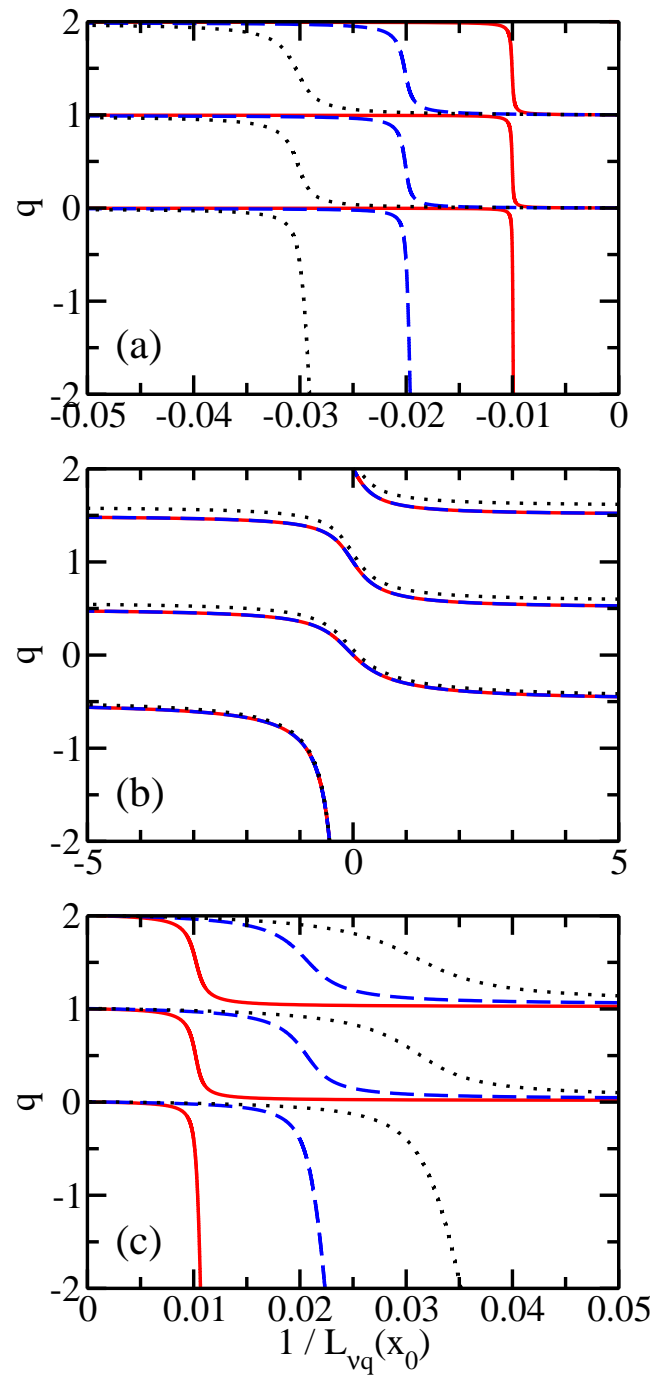

FIG. 3: (Color online) Non-integer quantum number $q$ as a function of $\left[L_{\nu q}\left(x_{0}\right)\right]^{-1}$ for (a) $s_{\nu}=3 / 5$, (b) $s_{\nu}=1 / 2$, and (c) $s_{\nu}=2 / 5$. In panels (a) and (c), the solid, dashed and dotted lines correspond to $x_{0}=1 \times 10^{-3}, 2 \times 10^{-3}$ and $3 \times$ $10^{-3}$, respectively. In panel (b), the solid, dashed and dotted lines correspond to $x_{0}=10^{-5}, 10^{-3}$ and $10^{-1}$, respectively; the solid and dashed lines are indistinguishable on the scale shown. Note that the scale of the $x$-axis is different in all three panels.

occur for negative $\left[L_{\nu q}\left(x_{0}\right)\right]^{-1}$ values with $\left|1 / L_{\nu q}\left(x_{0}\right)\right|$ small, where $q$ drops by roughly 1 over a small range of $\left[L_{\nu q}\left(x_{0}\right)\right]^{-1}$ values. The value of $\left|1 / L_{\nu q}\left(x_{0}\right)\right|$ at which $q$ drops decreases as $x_{0}$ decreases and reaches 0 in the ZR limit. The deviation of $q$ from $\approx 0,1, \cdots$ signals the existence of an $N$-body resonance, which arises when $F_{\nu q}(x)$ is fully determined by the irregular solution $g_{\nu q}(x)$. When only $g_{\nu q}(x)$ contributes, we find that the quantization condition becomes $q=-s_{\nu},-s_{\nu}+1, \cdots$ in the ZR limit, in agreement with note [43] of Ref. [42]. We refer to the corresponding energies as $E_{g, \nu q}$,

$$
E_{g, \nu q}=\left(2 q+s_{\nu}+1\right) \hbar \omega ; q=-s_{\nu},-s_{\nu}+1, \cdots .
$$

For $s_{\nu}=3 / 5, q$ equals $-3 / 5,2 / 5, \cdots$ on resonance and $\left|1 / L_{\nu q}\left(x_{0}\right)\right|=0$ for $x_{0} \rightarrow 0$.

The dependence of $q$ on the inverse logarithmic derivative $1 / L_{\nu q}\left(x_{0}\right)$ for $s_{\nu}=2 / 5$ [Fig. 3(c)] is similar to that for $s_{\nu}=3 / 5$ [Fig. 3(a)], with the main difference that $q$ now drops sharply for small positive $1 / L_{\nu q}\left(x_{0}\right)$ as opposed to negative $1 / L_{\nu q}\left(x_{0}\right)$. For $s_{\nu}=2 / 5, q$ equals $-2 / 5,3 / 5, \cdots$ on resonance and $\left|1 / L_{\nu q}\left(x_{0}\right)\right|=0$ for $x_{0} \rightarrow 0$. We note that the trapped $N$-body system supports a deep-lying bound state if the logarithmic derivative is negative [not shown in Fig. 33(c)].

The dependence of $q$ on $\left[L_{\nu q}\left(x_{0}\right)\right]^{-1}$ for $s_{\nu}=1 / 2$ [Fig. 3(b)] is distinctly different from that for $s_{\nu}=3 / 5$ and $2 / 5$. Figure 3 shows that the $q$ values depend much more weakly on $x_{0}$ for $s_{\nu}=1 / 2$ than for $s_{\nu} \neq 1 / 2$. Furthermore, $q$ approximately equals $0,1, \cdots$ for $1 / L_{\nu}\left(x_{0}\right)=$ 0 . As $\left|1 / L_{\nu q}\left(x_{0}\right)\right|$ increases, the $q$ values change gradually and approach half-integer values for large $\left|1 / L_{\nu q}\left(x_{0}\right)\right|$ (on resonance, $q=-1 / 2,1 / 2, \cdots$ ). In summary, for $s_{\nu} \neq 1 / 2$, the system exhibits an $N$-body resonance in the ZR limit for $1 / L_{\nu q}\left(x_{0}\right)=0$; for $q=-s_{\nu}$, e.g., the third term on the right hand side of Eq. (14) vanishes and $\lim _{x_{0} \rightarrow 0}\left|L_{\nu q}\left(x_{0}\right)\right|=\infty$. For $s_{\nu}=1 / 2$, in contrast, the system exhibits an $N$-body resonance in the ZR limit for $1 / L_{\nu q}\left(x_{0}\right)=\infty$; for $q=-s_{\nu}$, e.g., the first and the third term on the right hand side of Eq. (14) vanish and $\lim _{x_{0} \rightarrow 0} L_{\nu q}\left(x_{0}\right)=0$.

We now introduce a framework that expresses the resonance condition in terms of the generalized energydependent scattering length $\mathcal{V}_{s_{\nu}}(E)$. To define $\mathcal{V}_{s_{\nu}}(E)$, we consider the free-space system, i.e., we set the trapping frequency $\omega$ to 0 . Compared to the trapped system, only the hyperradial Schrödinger equation changes [see Eqs. (7) and (8)]. The hyperradial solution for the free-space system with positive energy $E$ can be written analogously to the solution for the trapped system [see Eq. (91)],

$$
\begin{array}{r}
F_{\nu k}(R)= \\
N_{\nu k}\left[R^{1 / 2} J_{s_{\nu}}(k R)-\tan \left(\delta_{s_{\nu}}(k)\right) R^{1 / 2} Y_{s_{\nu}}(k R)\right],
\end{array}
$$

where we use the continuous variable $k$ to label the solution of the hyperradial Schrödinger equation; $k$ is defined in terms of the $N$-body scattering energy $E$ and the hyperradial mass $\mu, k=\sqrt{2 \mu E / \hbar^{2}}$. In Eq. (16), $J_{s_{\nu}}$ and $Y_{s_{\nu}}$ denote the Bessel functions of the first and second kind, respectively. The phase shift $\delta_{s_{\nu}}(k)$ characterizes the $N$-body scattering process and can be used to define the generalized energy-dependent $N$-body scattering length $\mathcal{V}_{s_{\nu}}(k)$,

$$
\mathcal{V}_{s_{\nu}}(k)=-\frac{\tan \left(\delta_{s_{\nu}}(k)\right)}{k^{2 s_{\nu}}} \frac{2^{2 s_{\nu}} \Gamma\left(s_{\nu}\right) \Gamma\left(s_{\nu}+1\right)}{\pi} ;
$$


throughout this paper, the generalized energy-dependent scattering length is written, depending on the context, as $\mathcal{V}_{s_{\nu}}(k)$ or $\mathcal{V}_{s_{\nu}}(E)$. The power of $k$ in the denominator on the right hand side of Eq. (17) is chosen such that the generalized energy-independent $N$-body scattering length $\mathcal{V}_{s_{\nu}}(0), \mathcal{V}_{s_{\nu}}(0)=\lim _{k \rightarrow 0} \mathcal{V}_{s_{\nu}}(k)$, is well-behaved, i.e., such that $\mathcal{V}_{s_{\nu}}(k)$ approaches a constant in the $k \rightarrow 0$ limit. The "extra" factors on the right hand side of Eq. (17) are chosen such that $\mathcal{V}_{s_{\nu}}(0)$ reduces to $R_{0}^{2 s_{\nu}}$ if the boundary condition $F_{\nu k}\left(R_{0}\right)=0$ is imposed. We note that the definition of $\mathcal{V}_{s_{\nu}}(E)$, Eq. (17), has some similarities with that employed to describe the scattering of two particles with vanishing azimuthal quantum number in two spatial dimensions [66, 67]. For $s_{\nu}=1 / 2$, the $N$ body hyperradial scattering problem becomes formally identical to the scattering between two three-dimensional $s$-wave interacting particles and $\mathcal{V}_{s_{\nu}}(0)$ becomes formally equivalent to the usual three-dimensional $s$-wave scattering length $a_{s}$. In general, however, the generalized scattering length has units of (length) ${ }^{2 s_{\nu}}$.

To express the resonance condition for the trapped system in terms of the generalized energy-dependent scattering length $\mathcal{V}_{s_{\nu}}(E)$, we relate the eigenenergies of the trapped $N$-body system to $\mathcal{V}_{s_{\nu}}(E)$. To this end, we calculate the logarithmic derivative of the free-space solution $F_{\nu k}(R)$, Eq. (16), for small $x_{0}$. Considering $0<s_{\nu}<1$ and keeping terms up to order $x^{2 s_{\nu}-1}$, we find

$$
\begin{aligned}
L_{\nu k}\left(x_{0}\right) & \approx \frac{\frac{1}{2}-s_{\nu}}{x_{0}}-\frac{2^{s_{\nu}+1} s_{\nu} a_{\mathrm{ho}}^{2 s_{\nu}}}{\mathcal{V}_{s_{\nu}}(E)} x_{0}^{2 s_{\nu}-1}- \\
& \frac{2^{-s_{\nu}+1} \pi \cot \left(\pi s_{\nu}\right)}{\left[\Gamma\left(s_{\nu}\right)\right]^{2}}\left(\frac{E}{\hbar \omega}\right)^{s_{\nu}} x_{0}^{2 s_{\nu}-1} .
\end{aligned}
$$

For $s_{\nu}=1 / 2$, the first and third terms on the right hand side of Eq. (18) vanish and we find $L_{\nu k}\left(x_{0}\right) \approx$ $-\sqrt{2} a_{\mathrm{ho}} / \mathcal{V}_{s_{\nu}}(E)$ or $L_{\nu k}\left(R_{0}\right) \approx-\left[\mathcal{V}_{s_{\nu}}(E)\right]^{-1}$. To obtain an explicit relationship between the eigenenergies of the trapped $N$-body system and the generalized energydependent scattering length $\mathcal{V}_{s_{\nu}}(E)$ for $0<s_{\nu}<1$, we expand the logarithmic derivative of the trapped system, Eq. (14), up to order $x_{0}^{2 s_{\nu}-1}$ and set it equal to the logarithmic derivative of the free-space system [right hand side of Eq. (18)],

$$
\frac{a_{\mathrm{ho}}^{2 s_{\nu}}}{\mathcal{V}_{s_{\nu}}(E)}+h\left(E, s_{\nu}\right)=\frac{\Gamma\left(\frac{-E}{2 \hbar \omega}+\frac{1+s_{\nu}}{2}\right) \Gamma\left(1-s_{\nu}\right)}{\Gamma\left(\frac{-E}{2 \hbar \omega}+\frac{1-s_{\nu}}{2}\right) \Gamma\left(1+s_{\nu}\right) 2^{s_{\nu}}}
$$

where

$$
h\left(E, s_{\nu}\right)=\frac{\pi \cot \left(\pi s_{\nu}\right)}{2^{2 s_{\nu}} \Gamma\left(s_{\nu}\right) \Gamma\left(1+s_{\nu}\right)}\left(\frac{E}{\hbar \omega}\right)^{s_{\nu}} .
$$

It can be seen that the $1 / x_{0}$ divergencies are canceled and, furthermore, that Eq. (19) is - at this level of approximation -independent of $x_{0}$. Equation (19) determines the energy of the trapped $N$-body system in terms of the generalized energy-dependent scattering length $\mathcal{V}_{s_{\nu}}(E)$ and can be solved self-consistently for the eigenenergies $E$.

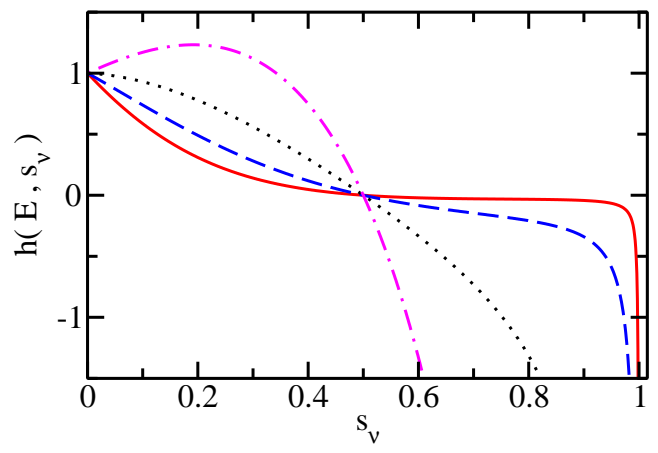

FIG. 4: (Color online) Solid, dashed, dotted and dash-dotted lines show the quantity $h\left(E, s_{\nu}\right)$, Eq. (20), as a function of $s_{\nu}$ for $E=1 \times 10^{-2} \hbar \omega, 1 \times 10^{-1} \hbar \omega, 1 \hbar \omega$, and $10 \hbar \omega$, respectively.

For $s_{\nu}=1 / 2, h\left(E, s_{\nu}\right)$ vanishes and $\Gamma\left(1-s_{\nu}\right) /[\Gamma(1+$ $\left.\left.s_{\nu}\right) 2^{s_{\nu}}\right]$ reduces to $\sqrt{2}$. Setting $\left[L_{\nu q}\left(x_{0}\right)\right]^{-1}$ equal to $-\mathcal{V}_{s_{\nu}}(E) /\left(\sqrt{2} a_{\mathrm{ho}}\right)$ [see discussion after Eq. (18)], Fig. 3(b) can be interpreted as showing the non-integer quantum number $q$ of the trapped $N$-body system as a function of $-\mathcal{V}_{s_{\nu}}(E) /\left(\sqrt{2} a_{\mathrm{ho}}\right)$. A vanishing generalized energy-dependent scattering length implies an infinitely large logarithmic derivative, and an infinitely large generalized energy-dependent scattering length implies a vanishing logarithmic derivative. From Eq. (19), it follows for $s_{\nu}=1 / 2$ that $E=E_{g, \nu q}$ if $\mathcal{V}_{s_{\nu}}(E)$ diverges, i.e., the $N$-body resonance occurs when $\left[\mathcal{V}_{s_{\nu}}(E)\right]^{-1}$ vanishes. The analysis outlined here for $s_{\nu}=1 / 2$ is formally identical to that of the trapped two-particle system. Identifying $\mathcal{V}_{s_{\nu}}(E)$ with the $s$-wave atom-atom scattering length, Eq. (19) is identical to the well-known eigenequation for two trapped $s$-wave interacting particles with ZR interactions [54].

For $s_{\nu} \neq 1 / 2$ and $E>0, h\left(E, s_{\nu}\right)$ does not vanish and introduces an additional energy dependence on the left hand side of Eq. (19), which originates from the explicit energy-dependence of the logarithmic derivative of the free-space solution. If we artificially set $h\left(E, s_{\nu}\right)$ to zero, the eigenenergy $E$ equals $E_{g, \nu q}$ when $\mathcal{V}_{s_{\nu}}(E)$ diverges. Inclusion of $h\left(E, s_{\nu}\right)$ shifts the energy for all $\mathcal{V}_{s_{\nu}}(E) \neq 0$ $\left(s_{\nu} \neq 1 / 2\right)$ and modifies the resonance condition. In particular, the resonance condition becomes

$$
-a_{\mathrm{ho}}^{2 s_{\nu}} / \mathcal{V}_{s_{\nu}}\left(E_{g, \nu q}\right)=h\left(E_{g, \nu q}, s_{\nu}\right) .
$$

Figure 4 shows $h\left(E, s_{\nu}\right)$ as a function of $s_{\nu}$ for four different energies, i.e., $E=10^{-2} \hbar \omega-10 \hbar \omega . h\left(E, s_{\nu}\right)$ vanishes if $s_{\nu}$ equals $1 / 2$ (see also above), and increases with increasing $\left|s_{\nu}-1 / 2\right|$. For $E>0, h\left(E, s_{\nu}\right)$ takes on negative values for $1 / 2<s_{\nu}<1$ and positive values for $0<s_{\nu}<1 / 2$. As can be seen, $h\left(E, s_{\nu}\right)$ approaches $-\infty$ as $s_{\nu}$ approaches 1 . Thus, for large $\left|\mathcal{V}_{s_{\nu}}(E) / a_{\text {ho }}^{2 s_{\nu}}\right|$ and $s_{\nu} \neq 1 / 2$, the $a_{\mathrm{ho}}^{2 s_{\nu}} / \mathcal{V}_{s_{\nu}}(E)$ term is small and the $h\left(E, s_{\nu}\right)$ term dominates the left hand side of Eq. (19). 

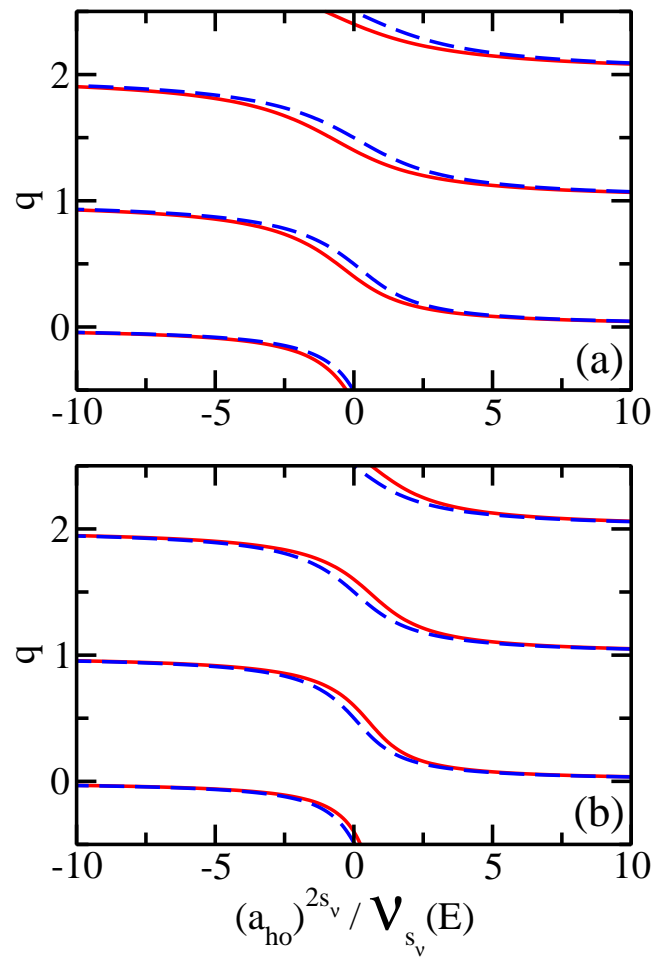

FIG. 5: (Color online) Dashed lines show the non-integer quantum number $q$ of the trapped $N$-body system, obtained by solving Eq. (19) self-consistently, as a function of the generalized energy-dependent scattering length $\mathcal{V}_{s_{\nu}}(E)$ for (a) $s_{\nu}=3 / 5$ and (b) $s_{\nu}=2 / 5$. For comparison, solid lines show the $q$ values that result when $h\left(E, s_{\nu}\right)$ is artificially set to zero.

For small $\left|\mathcal{V}_{s_{\nu}}(E) / a_{\text {ho }}^{2 s_{\nu}}\right|$, in contrast, the $a_{\text {ho }}^{2 s_{\nu}} / \mathcal{V}_{s_{\nu}}(E)$ term is large and the $h\left(E, s_{\nu}\right)$ term on the left hand side of Eq. (19) can be neglected. Figures 5 (a) and (b) show the eigenenergy of the trapped system as a function of the generalized energy-dependent scattering length $\mathcal{V}_{s_{\nu}}(E)$ for $s_{\nu}=3 / 5$ and $2 / 5$, respectively. Dashed lines show the non-integer quantum number $q$ obtained by solving Eq. (19) self-consistently while solid lines show the noninteger quantum number $q$ that results when $h\left(E, s_{\nu}\right)$ is artificially set to 0 . Figure 5 shows that the inclusion of the $h\left(E, s_{\nu}\right)$ term shifts the eigenenergies up (down) compared to those calculated for $h\left(E, s_{\nu}\right)=0$ for $s_{\nu}=3 / 5$ $\left(s_{\nu}=2 / 5\right)$. The term $h\left(E, s_{\nu}\right)$ has a small effect when $\left|\mathcal{V}_{s_{\nu}}(E)\right|$ is small but introduces a notable shift of the energies when $\left|\mathcal{V}_{s_{\nu}}(E)\right|$ is large.

As discussed after Eq. (11), the ZR framework employed in this section does not allow for the determination of the actual value of the logarithmic derivative or, equivalently, the generalized scattering length. Let us imagine that the generalized scattering length $\mathcal{V}_{s_{\nu}}(E)$ can be controlled experimentally by tuning the interactions between like atoms [4] or by varying the parame- ters of a lattice confinement [44]. The realization of an $N$-body resonance for $s_{\nu} \neq 1 / 2$ requires extreme finetuning since both $h\left(E, s_{\nu}\right)$ and $\mathcal{V}_{s_{\nu}}(E)$ vary with energy. On the other hand, for $s_{\nu}=1 / 2$, only $\mathcal{V}_{s_{\nu}}(E)$ varies with energy. The energy-dependence of $\mathcal{V}_{s_{\nu}}(E)$ is expected to be weakest for $s_{\nu}=1 / 2$ since the effective angular momentum barrier in the $R$ coordinate vanishes in this case. This reasoning is motivated by what is known from "ordinary" two-particle scattering (see, e.g., Ref. 51]): In general, two-particle $s$-wave scattering (no angular momentum barrier) exhibits a much weaker energy-dependence than two-particle $p$ - or $d$-wave scattering (finite angular momentum barrier). We conclude that it should be more likely to realize an $N$-body resonance if $s_{\nu}=1 / 2$ than if $s_{\nu} \neq 1 / 2$.

Lastly, we note that application of the two-body scattering framework to the hyperradial problem at hand implies that the free-space system supports an $N$-body bound state with zero energy when $\mathcal{V}_{s_{\nu}}(0)$ diverges. Furthermore, the $N$-body system supports a single weaklybound free-space bound state when $\mathcal{V}_{s_{\nu}}(E)$ is large and positive, and no weakly-bound free-space bound state when $\mathcal{V}_{s_{\nu}}(E)$ is negative.

The following section introduces the stochastic variational (SV) approach which we use to numerically solve the Schrödinger equation in the relative coordinates for few-body systems interacting through FR potentials. This approach employs Jacobi coordinates instead of hyperspherical coordinates and makes no assumption about the small $R$ behavior of $F_{\nu q}(R)$ : the small $R$ behavior is not treated as input but instead is a natural part of the solution. Connections between the results based on the approaches discussed in Secs. $\amalg \mathrm{IB}$ and $\amalg \mathrm{IC}$ will be made in Sec. III.

\section{Stochastic variational treatment}

The SV approach [68 70] expands the relative wave function $\Psi$ in terms of basis functions $\varphi_{k}$,

$$
\Psi=\sum_{k=1}^{N_{\text {basis }}} c_{k} \mathcal{A}\left[\varphi_{k}(\vec{x})\right],
$$

where the $c_{k}$ denote expansion coefficients, $\mathcal{A}$ denotes an operator that ensures the anti-symmetry of the basis functions, and $\vec{x}$ collectively denotes the Jacobi vectors $\vec{\rho}_{j}$, where $j=1, \cdots, N-1$. The basis functions [see Eqs. (23) and (25) for their explicit forms] are chosen such that the Hamiltonian matrix can be constructed analytically. The eigenenergies $E$ of the relative Hamiltonian, which provide an upper bound to the exact eigenenergies, are then obtained by diagonalizing the Hamiltonian matrix. The variational bound can be improved systematically by increasing the size of the basis set (i.e., by increasing $N_{\text {basis }}$ ) and by varying the parameters $\vec{u}^{(k)}$ and $d_{i j}^{(k)}$ [see Eq. (23)] or $\vec{s}^{(k)}$ and $d_{i j}^{(k)}$ [see Eq. (25)]. Our primary interest in this work is to describe the energetically 
lowest-lying state of various $\left(N_{1}, N_{2}\right)$ systems. The energetically lowest lying state of the $(2,1)$ system, e.g., has natural parity (see Fig. 2) while that of the $(3,1)$ system has unnatural parity. Thus, we need to consider basis functions that can describe both natural and unnatural parity states.

To treat natural parity states, i.e., states with $\Pi=$ $(-1)^{L}$, we employ basis functions that are written in terms of the spherical harmonic $Y_{L 0}$, which determines the relative orbital angular momentum of the system and depends on the unit vector $\hat{v}^{(k)}$ [68],

$$
\varphi_{k}(\vec{x})=\left|\vec{v}^{(k)}\right|{ }^{L} Y_{L 0}\left(\hat{v}^{(k)}\right) \exp \left[-\sum_{i<j}^{N}\left(\frac{r_{i j}}{\sqrt{2} d_{i j}^{(k)}}\right)^{2}\right],
$$

where

$$
\vec{v}^{(k)}=\sum_{j=1}^{N-1} u_{j}^{(k)} \vec{\rho}_{j}
$$

Here, the $u_{j}^{(k)}$ form a $(N-1)$-dimensional parameter vector that determines how the relative orbital angular momentum $L$ of the $\left(N_{1}, N_{2}\right)$ system is distributed among the $(N-1)$ Jacobi vectors $\vec{\rho}_{j}$. We find that the optimal set of widths $d_{i j}^{(k)}$ depends quite strongly on the mass ratio $\kappa$. For the $(2,1)$ system with equal-masses, e.g., threebody bound states are absent [35, 71]. This implies that three-body correlations are largely absent, and that the contribution of basis functions with more than one $d_{i j}^{(k)}$ of the order of the range of the underlying two-body potential contribute negligibly to the wave function [60, 70]. When $\kappa \approx 12$, in contrast, three-body correlations are non-negligible and basis functions $\varphi_{k}$ that are characterized by three widths $d_{i j}^{(k)}$ of the order of the range of the two-body potential contribute notably [48].

To describe states with unnatural parity, we employ socalled geminal-type basis functions $\varphi_{k}$ that are neither eigenfunctions of the angular momentum operator nor the parity operator 68,72 ,

$$
\varphi_{k}(\vec{x})=\exp \left[-\sum_{i<j}^{N}\left(\frac{r_{i j}}{\sqrt{2} d_{i j}^{(k)}}\right)^{2}+\left(\vec{s}^{(k)}\right)^{T} \vec{x}\right] .
$$

Here, $\left(\vec{s}^{(k)}\right)^{T} \vec{x}$ is just the dot product between two $3(N-1)$ dimensional vectors. The $3(N-1)$-dimensional parameter vector $\vec{s}^{(k)}$ is, together with the $N(N-1) / 2$ widths $d_{i j}^{(k)}$, optimized semi-stochastically for each basis function $\varphi_{k}$. In general, the determination of the natural parity states [see Eq. (23)] is, for the same $N$, numerically significantly more efficient than that of unnatural parity states [see Eq. (25)].

The SV approach also allows for the determination of structural properties. To calculate structural properties, we follow two different approaches [73]: (i) We implement the analytically known matrix elements 68] for the operator $A$ of interest, and calculate the expectation value of $A$ using the known $c_{k}$, where $k=1, \cdots, N_{\text {basis. }}$ (ii) We calculate the expectation value of the operator $A$ by sampling the normalized density $|\psi|^{2}$ through Metropolis sampling [74]. In the limit of infinitely many Monte Carlo samples, the results of approach (ii) should agree with those of approach (i). Where possible, we have used this to check our implementations. To calculate the hyperradial density $P_{\text {hyper }}(R)$, where

$$
\int_{0}^{\infty} P_{\text {hyper }}(R) d R=1,
$$

we employ approach (ii). The pair distribution functions $P_{\mathrm{hl}}(r)$ and $P_{\mathrm{hh}}(r)$ for heavy-light and heavy-heavy atom pairs, normalized such that

$$
4 \pi \int_{0}^{\infty} P_{\mathrm{hl}}(r) r^{2} d r=1
$$

[and similarly for $P_{\mathrm{hh}}(r)$ ], is calculated using approach (i) for the wave function written in terms of the basis functions given in Eq. (23) and approach (ii) for that written in terms of the basis functions given in Eq. (25).

\section{RESULTS}

This section presents our results from the SV calculations for FR interactions and interprets our findings within the hyperspherical framework. Sections IIIB present the energetics and structural properties for the $(2,1)$ and $(3,1)$ systems, respectively.

\section{A. Three-body resonances}

We start our discussion with the infinitely strongly interacting $(2,1)$ system at unitarity, and then discuss the behavior away from the two-body resonance. Since the $L^{\Pi}=1^{-}$state is, as discussed in Sec. IIB, the energetically lowest lying state for all $\kappa$ of interest, we restrict our SV calculations to this symmetry. For each mass ratio $\kappa$, we consider different $r_{0}$ with $r_{0} \ll a_{\text {ho }}$. Symbols in Figs. [ and 7 show examples for the ground state energy of systems with $\kappa=1$ through 12.316 interacting through $V_{\mathrm{g}}$. By fitting the range-dependent $\mathrm{SV}$ energies to simple three- to five-parameter expressions, we extrapolate the FR SV energies to the $r_{0} \rightarrow 0$ limit. For $\kappa=1$ and 6 (circles and squares in Fig. 6), the SV energies approach the ZR limit approximately linearly from above and below, respectively. For $\kappa=10$, the FR energies are best described by a quadratic three-parameter fit. As $\kappa$ increases further, the range-dependence increases notably (see circles and squares in Fig. 7 for $\kappa=12.25$ and 12.3). For $\kappa=12.3131$ and 12.314 (diamonds and triangles in Fig. 7), in contrast, the range-dependence is comparatively small and the SV energies approach the ZR limit approximately linearly from above. Finally, for $\kappa=12.316$ (crosses in Fig. 17) the energies decrease as 


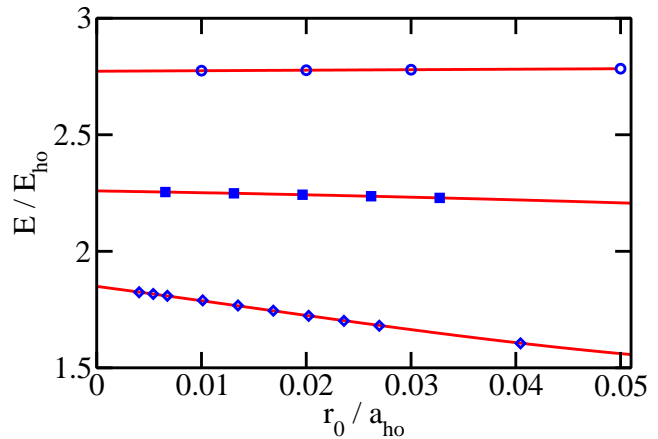

FIG. 6: (Color online) Circles, squares and diamonds show the SV energies for the $(2,1)$ system with $L^{\Pi}=1^{-}$at unitarity for $\kappa=1,6$ and 10, respectively. The unlike particles interact through $V_{\mathrm{g}}$. Solid lines show three-parameter fits of the form $\sum_{j=0}^{2} c_{j} r_{0}^{j}$ to the $\mathrm{SV}$ energies.

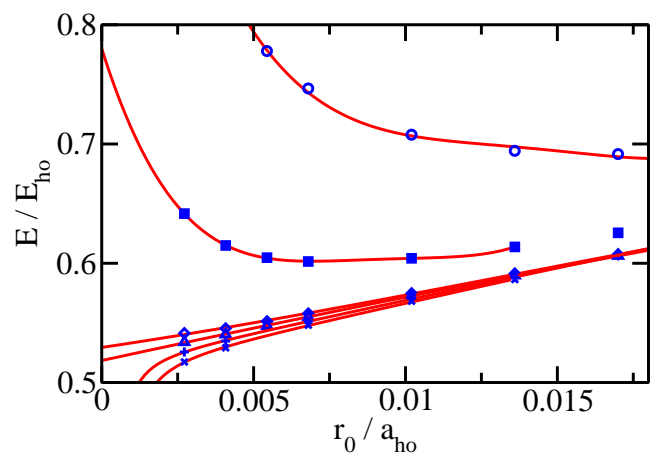

FIG. 7: (Color online) Circles, squares, diamonds, triangles, pluses and crosses show the SV energies for the $(2,1)$ system with $L^{\Pi}=1^{-}$at unitarity for $\kappa=$ $12.25,12.3,12.3131,12.314,12.315$ and 12.316 , respectively. The unlike particles interact through $V_{\mathrm{g}}$. Solid lines show three- to five-parameter fits to the SV energies.

$1 / r_{0}^{2}$ for sufficiently small $r_{0}$, indicating the presence of a three-body bound state. For yet larger $\kappa$ (not shown in Fig. 7), the energy of the trapped system becomes negative. The appearance of the three-body bound state indicates the presence of a three-body resonance. We estimate the resonance for the potential $V_{\mathrm{g}}$ in the $r_{0} \rightarrow 0$ limit to be located at $\kappa \approx 12.314(2)$. The uncertainty in our estimate for the resonance position arises mainly from the extrapolation of our SV energies to the ZR limit. The SV energies for $\kappa=12.315$, e.g., are nearly equally well described by fits of the form $c_{-2} / r_{0}^{2}+c_{-1} / r_{0}+c_{0}$ (shown in Fig. 17) and $c_{0}+c_{1} r_{0}+c_{2} r_{0}^{2}$ (not shown in Fig. (7).

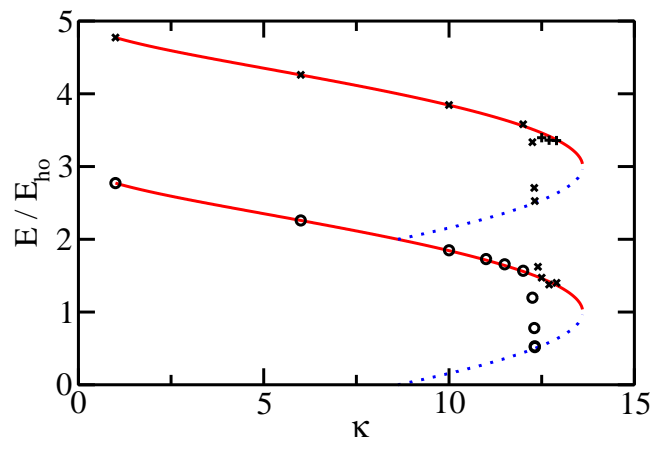

FIG. 8: (Color online) Energies of the $(2,1)$ system with $L^{\Pi}=1^{-}$at unitarity. Circles, crosses and pluses show the $\mathrm{SV}$ energies for $V_{\mathrm{g}}$, extrapolated to the ZR limit, for the three energetically lowest-lying states as a function of $\kappa$. Solid and dotted lines show $E_{f, 0 q}(q=0$ and 1$)$ and $E_{g, 0 q}\left(q=-s_{0}\right.$ and $\left.-s_{0}+1\right)$, respectively. This figure has been adapted from Ref. [48].

Symbols in Fig. 8 show the extrapolated ZR energies for the three energetically lowest lying states of the $(2,1)$ system with $L^{\Pi}=1^{-}$interacting through $V_{\mathrm{g}}$ with $1 / a_{s}=0$. The dropping of the energies around $\kappa \approx 12.3$ associated with the three-body resonance is clearly visible: The ground state energy (circles) becomes negative while the energies of the first excited state (crosses) and the second excited state (pluses) drops by approximately $2 \hbar \omega$. For comparison, solid and dotted lines in Fig. 8 show the ZR energies $E_{f, 0 q}$ [Eq. (11) with $q=0$ and 1] and $E_{g, 0 q}$ [Eq. (15) with $q=-s_{0}$ and $-s_{0}+1$ ], respectively. Away from the three-body resonance, the extrapolated ZR energies agree well with $E_{f, 0 q}$. On resonance, the extrapolated ZR energies agree well with $E_{g, 0 q}$. Our analysis is fully consistent with the general discussion of Ref. [42], where it was found that universal states for systems under spherically symmetric harmonic confinement must have an energy larger than $1 \hbar \omega$ and that states with energy less than $1 \hbar \omega$ are necessarily non-universal. The admixture of the irregular solution $g_{\nu q}$ requires that the boundary condition of the hyperradial wave function be specified, which makes the system properties dependent on an additional parameter and thus non-universal. It has been pointed out previously [42] that the threebody system is again scale-invariant at the three-body resonance. Our work (see also Ref. [48]) shows an example for this non-universal three-body resonance. This non-trivial three-body resonance has also recently been investigated by Gandolfi and Carlson [49], who studied the free-space problem.

The mass ratio at which the three-body resonance exists depends on the details of the underlying two-body potential. As has been argued in Ref. 48] and in Sec. IIB. it seems most likely that the three-body resonance occurs 

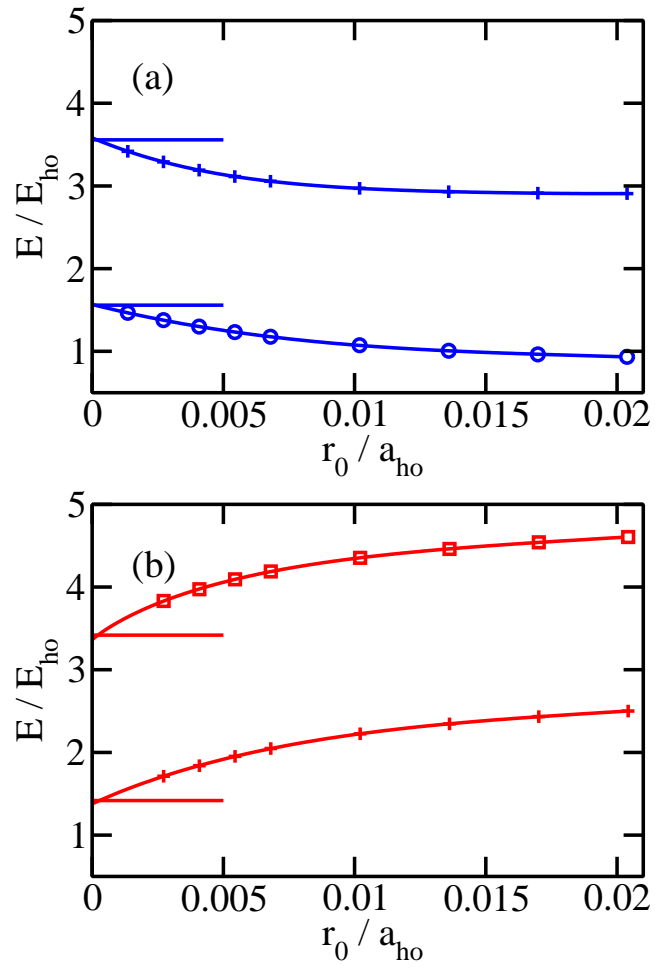

FIG. 9: (Color online) Symbols show the SV energies for the $(2,1)$ system with $L^{\Pi}=1^{-}$interacting through $V_{\mathrm{g}}$ at unitarity for (a) $\kappa=12$ and (b) $\kappa=12.7$, respectively, as a function of $r_{0}$. For $\kappa=12$, the energies of the ground state (circles) and first excited state (pluses) are shown. For $\kappa=12.7$, the energies of the first and second excited states (pluses and squares) are shown. Solid lines show fits to the SV energies. Horizontal lines show the energy $E_{f, 0 q}$ for $q=0$ and 1 .

when the effective hyperangular momentum barrier vanishes, i.e., when $s_{\nu} \approx 1 / 2$. For the $(2,1)$ system with ZR interactions and $L^{\Pi}=1^{-}$symmetry, this is the case when $\kappa \approx 12.3131$ (see Fig. 22); this value is close to the resonance position found for the purely attractive Gaussian potential $V_{\mathrm{g}}$. In our example, the trimer on resonance is large [this follows from Eq. (12)]; this implies a long lifetime and thus opens the intriguing possibility of studying trimer correlations in many-body systems.

Symbols in Figs. 9(a) and (b) shows the SV energies for the $(2,1)$ system with $\kappa=12$ and 12.7 , respectively, interacting through $V_{\mathrm{g}}$ with $1 / a_{s}=0$ as a function of $r_{0}$. For both $\kappa$, the SV energies for the "lowest universal state" and the "second lowest universal state" are shown [for $\kappa=12.7$, the ground state (not shown) corresponds to a tightly bound non-universal trimer]. The extrapolated ZR energies of the two universal states agree well with the energies $E_{f, 0 q}(q=0$ and 1$)$, which are shown by horizontal lines. This implies that the $2 \hbar \omega$ spacing expected for universal states [42] is fullfilled with good accuracy. For $\kappa=12$ and 12.7, our fits result in an energy spacing of $2.014 \hbar \omega$ and $1.984 \hbar \omega$, respectively. For finite $r_{0}$, the spacing between the two lowest universal states deviates from the $2 \hbar \omega$ spacing. For $r_{0} \approx 0.01 a_{\mathrm{ho}}$, e.g., we find a spacing of $\approx 1.90 \hbar \omega$ and $2.13 \hbar \omega$ for $\kappa=12$ and 12.7 , respectively.

To make an explicit connection between the FR SV energies and the energies obtained within the hyperspherical framework (see Sec. IIB), we assume a direct proportionality between the range $r_{0}$ of the two-body interaction potential and the hyperradius $R_{0}$ at which the logarithmic derivative of the hyperradial function $F_{\nu q}(R)$ is imposed. The proportionality factor between $r_{0}$ and $R_{0}$ cannot be determined within the model; we find that a proportionality factor of order 5 to 10 , i.e., $r_{0} \approx 5-10 R_{0}$, is appropriate. Figures 10(a)-(d) exemplarily show the eigenenergies predicted by the hyperspherical framework as a function of $R_{0}$ for $s_{\nu} \approx 0.5579$ and the lowest allowed $q$ value, for $s_{\nu}=1 / 2$ and the lowest allowed $q$ value, and for $s_{\nu}=0.4180$ and the lowest and second lowest allowed $q$ values for various values of the logarithmic derivative. For the $(2,1)$ system with $L^{\Pi}=1^{-}$symmetry, these $s_{\nu}$ values correspond to $\kappa=12,12.3131$ and 12.7 , respectively. We find that there exists a value of the logarithmic derivative for each $\kappa$ that predicts energies as a function of $R_{0}$ [via Eq. (14)] that are in qualitative agreement with the dependence of the SV energies on $r_{0}$. For example, the SV energies of the lowest universal state for $\kappa=12.7$ approach the ZR limit from above [pluses in Fig. 9(b)], in agreement with the dependence of the energies obtained within the hyperspherical framework on $R_{0}$ [see Fig. 10(d)]. For $\kappa=12$, in contrast, the SV energies of the ground state approach the ZR limit from below [circles in Fig. 9(a)], in agreement with the dependence of the energies obtained within the hyperspherical framework on $R_{0}$ for small $\left|L_{\nu q}\right|$ [see Fig. [10(a)]. Although the comparison between the SV energies and those obtained within the hyperspherical framework leads to a consistent picture (including the existence or absence of bound states), the analysis unfortunately does not allow for the unambiguous extraction of the value of the logarithmic derivative or the generalized scattering length.

To gain additional insight into the three-particle system, we analyze the structural properties of the $(2,1)$ system interacting through $V_{\mathrm{g}}$ with $L^{\Pi}=1^{-}, 1 / a_{s}=0$ and $r_{0}=0.003 a_{\mathrm{ho}}$. Figure 11] shows the scaled pair distribution function $P_{\mathrm{hl}}(r) r^{2}$ for the heavy-light pairs for $\kappa=1$ through 12.5, while Fig. 12 shows the scaled pair distribution function $P_{\mathrm{hh}}(r) r^{2}$ for the heavy-heavy pair for the same mass ratios. As $\kappa$ increases, the amplitudes of $P_{\mathrm{hl}}(r) r^{2}$ and $P_{\mathrm{hh}}(r) r^{2}$ increase at small distances and decrease at large distances. The scaled pair distribution function for the spin-up - spin-down distance coordinate [75] for $\kappa=1$ [dash-dotted lines in Fig. 11(a)], e.g., shows a "two-bump structure" that has been previously interpreted within an atom-dimer picture [60]: The "bump" at smaller $r, r \approx 0.1 a_{\mathrm{ho}}$, reflects the formation of a dimer while the "bump" at larger $r, r \approx 1.5 a_{\mathrm{ho}}$, 


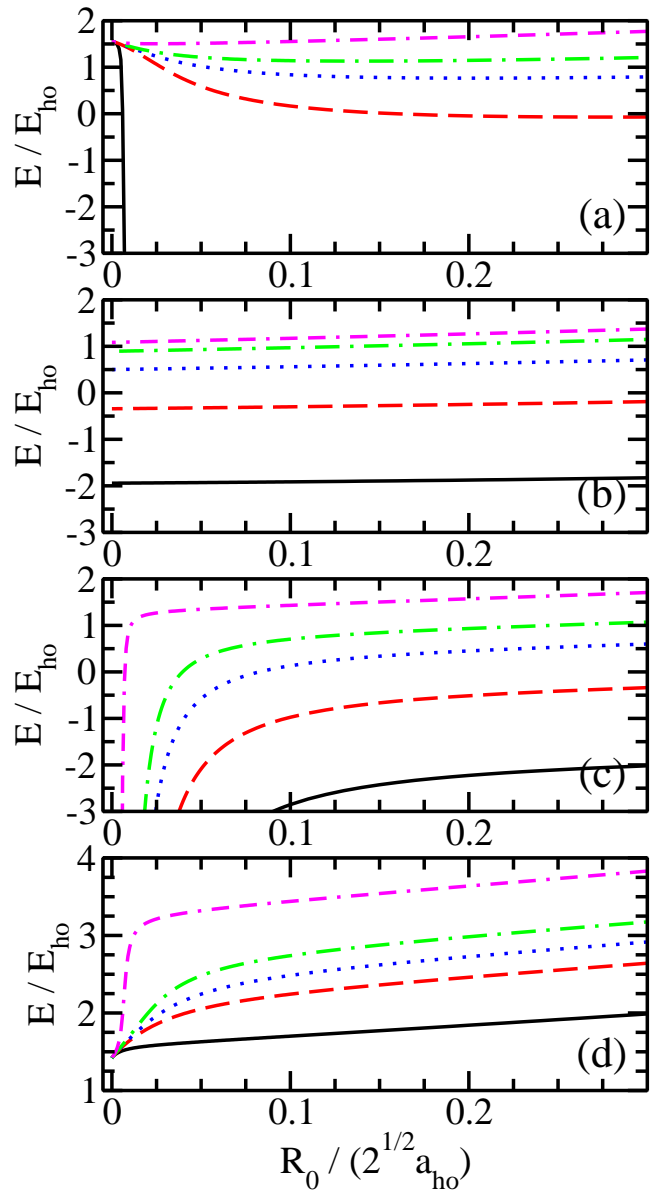

FIG. 10: (Color online) Eigenenergy $E$ of the trapped system at unitarity, obtained from Eq. (14), as a function of $R_{0}$ for (a) $s_{\nu} \approx 0.5579$ (lowest allowed $q$ value), (b) $s_{\nu}=1 / 2$ (lowest allowed $q$ value), (c) $s_{\nu} \approx 0.4180$ (lowest allowed $q$ value), and (d) $s_{\nu} \approx 0.4180$ (second lowest allowed $q$ value). Dash-dash-dotted, dash-dotted, dotted, dashed and solid lines correspond to (a) $L_{\nu q}\left(x_{0}\right)=10,1,0,-1$, and -10 , (b) $L_{\nu q}\left(x_{0}\right)=2,1,0,-1$, and -2 , (c) $L_{\nu q}\left(x_{0}\right)=10,1,0,-1$, and -2 , and (d) $L_{\nu q}\left(x_{0}\right)=10,1,0,-1$, and -10 .

reflects the fact that the "spare" spin-up atom sits further away from the spin-down atom than the spin-up atom that forms the dimer. For $\kappa=6.7$ [dotted lines in Figs. 11(a) and 12(a)], which corresponds to the ${ }^{40} \mathrm{~K}$ ${ }^{6} \mathrm{Li}$ mixture, the heavy-light and heavy-heavy pair distribution functions reflect the effective attraction between the two heavy atoms. Compared to the $\kappa=1$ system, the likelihood of finding the two like atoms at distances smaller than $a_{\text {ho }}$ is significantly increased. For $\kappa=12.5$, both the scaled heavy-light and heavy-heavy pair distribution functions fall off approximately exponentially at length scales smaller than $a_{\text {ho }}$, as expected for a threebody bound state.

Symbols in Fig. 13 show the hyperradial density
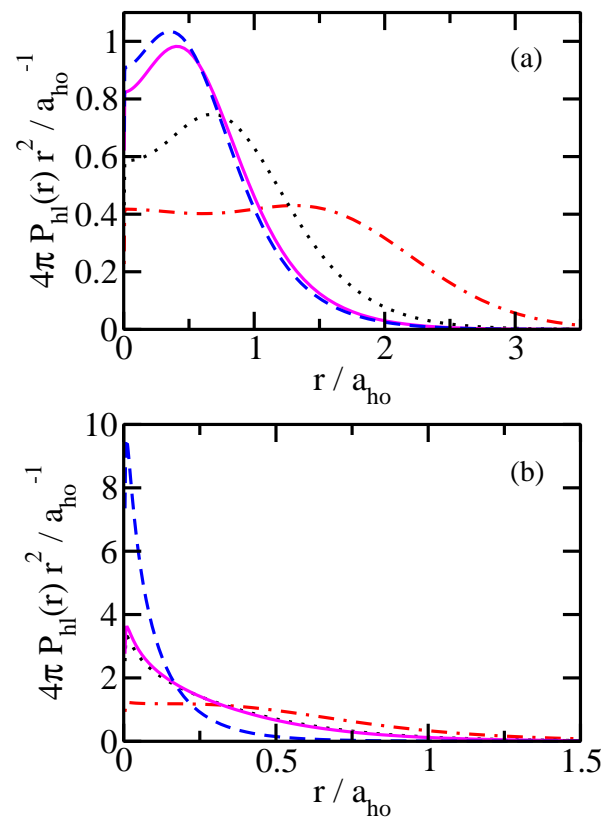

FIG. 11: (Color online) Dash-dotted, dotted, solid, and dashed lines show the scaled pair distribution function $P_{\mathrm{hl}}(r) r^{2}$ for the heavy-light pair of the $(2,1)$ system at unitary with $L^{\Pi}=1^{-}$for (a) $\kappa=1,6.7,11$, and 11.5 , and (b) $\kappa=12,12.3,12.314$, and 12.5 , respectively. The heavy-light particles interact through $V_{\mathrm{g}}(r)$ with $r_{0}=0.003 a_{\text {ho }}$. Note the different scales of the axis in panels (a) and (b).

$P_{\text {hyper }}(R)$ for the FR interaction $V_{\mathrm{g}}$ with $r_{0}=0.003 a_{\mathrm{ho}}$ and $1 / a_{s}=0$ for various $\kappa$. For comparison, lines show the hyperradial densities $\left|F_{\nu q}(R)\right|^{2}$ obtained from the ZR model. As can be seen from Eq. (12), $F_{\nu q}(R)$ depends on $s_{\nu}$ and $q$. For all $\kappa$ considered in Fig. 13, we use the $s_{0}$ obtained by solving the hyperangular Schrödinger equation for ZR interactions. The quantum number $q$ is set to zero for $\kappa=1$ and 6.7 [see Eq. (11)]. For $\kappa>8.619$, we adjust the short-range boundary condition of $F_{\nu q}(R)$ so as to reproduce the FR energies, i.e., we calculate $q$ according to Eq. (10) with $E$ taken from the FR SV calculation. Figure 13 shows good agreement between the hyperradial densities obtained from the SV calculations and those obtained within the ZR model. For $\kappa=12$, e.g., the admixture of the irregular solution $g_{0 q}$ is clearly reflected in the hyperradial density: Using $q=0$, i.e., using $f_{0 q}$ only, results in a notably poorer description of the system (not shown).

So far, we have considered systems with infinitely large two-body $s$-wave scattering length. To shed further light on the $(2,1)$ system in the vicinity of the three-body resonance, we also performed calculations for finite $a_{s}$. For the free-space $(2,1)$ system with $L^{\Pi}=1^{-}$and positive $a_{s}$, a single universal three-body bound state whose energy is a few times that of the two-body system has 

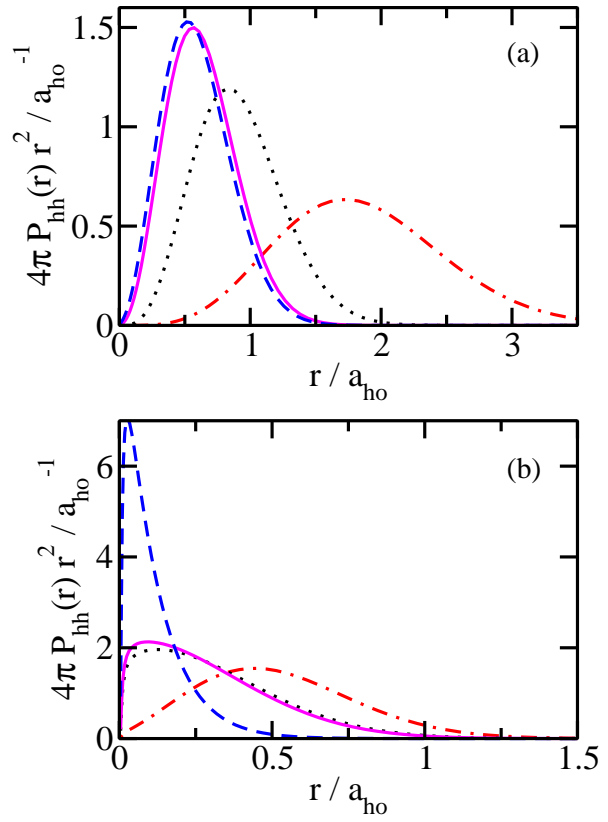

FIG. 12: (Color online) Dash-dotted, dotted, solid, and dashed lines show the scaled pair distribution function $P_{\mathrm{hh}}(r) r^{2}$ for the heavy-heavy pair of the $(2,1)$ system at unitary with $L^{\Pi}=1^{-}$for (a) $\kappa=1,6.7,11$, and 11.5, and (b) $\kappa=12,12.3,12.314$, and 12.5 , respectively. The heavy-light particles interact through $V_{\mathrm{g}}(r)$ with $r_{0}=0.003 a_{\text {ho }}$. Note the different scales of the axis in panels (a) and (b).

been predicted to exist for $\kappa \approx 8.173$ to 12.917 , and two universal bound states have been predicted to exist for $\kappa \approx 12.917$ to 13.606 [46]. The three-body bound states discussed here for the trapped system are distinctly different from these universal states, i.e., they do not approach those discussed in Ref. [46] when $\omega$ approaches 0 . Symbols in Fig. 14 show the energies of the trapped three-body system as a function of $1 / a_{s}$ for the interaction potential $V_{\mathrm{g}}$ with $r_{0}=0.005 a_{\text {ho }}$ for three different mass ratios $\kappa, \kappa=12$ (below the three-body resonance), $\kappa=12.314$ (at the three-body resonance), and $\kappa=12.5$ (above the three-body resonance). We note that the three-body energies depend strongly on $r_{0}$. As expected, for negative (positive) $a_{s}$, the three-body energy lies above (below) the corresponding energy at unitarity. For comparison, a solid line shows the energy of the trapped two-body system with ZR interactions. For small $\left|a_{s}\right|\left(a_{s}>0\right)$, the two-body energy varies approximately as $1 / a_{s}^{2}$. To obtain a semi-quantitative description of the three-body energies on the positive scattering length side, we write $E / E_{\mathrm{ho}}=c_{1}\left(a_{\mathrm{ho}} / a_{s}-c_{3}\right)^{c_{2}}$. Fitting our three-body energies for $a_{\mathrm{ho}} / a_{s}>0.5$, we find that the $c_{2}$ coefficient changes from 1.65 over 1.55 to 1.52 as $\kappa$ changes from 12 over 12.314 to 12.5 . The resulting fits are shown by dotted lines in Fig. 14.

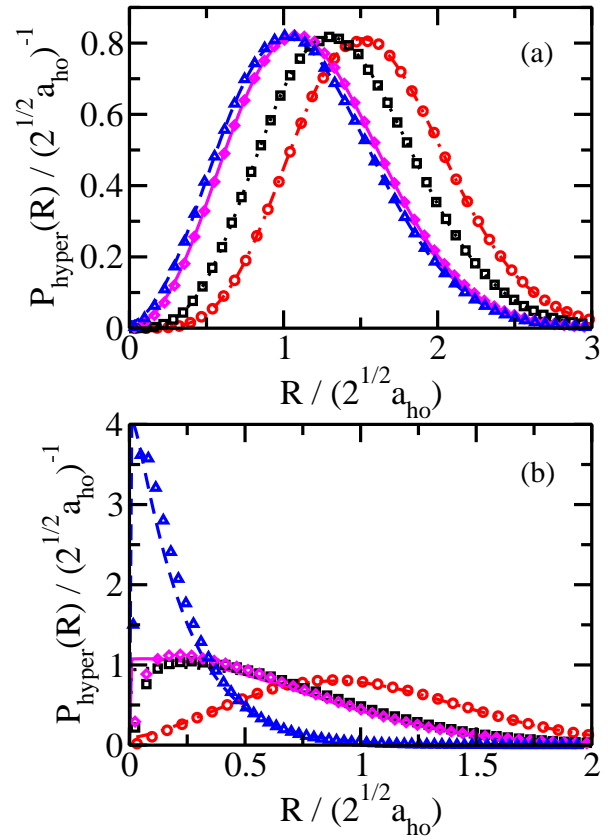

FIG. 13: (Color online) Hyperradial density $P_{\text {hyper }}(R)$ of the $(2,1)$ system with $L^{\Pi}=1^{-}$at unitary. Symbols show the results from the SV calculation where the heavy-light particles interact through $V_{\mathrm{g}}(r)$ with $r_{0}=0.003 a_{\text {ho }}$ for (a) $\kappa=1$ (circles), $\kappa=6.7$ (squares), $\kappa=11$ (diamonds), and $\kappa=11.5$ (triangles), and (b) $\kappa=12$ (circles), $\kappa=12.3$ (squares), $\kappa=12.314$ (diamonds), and $\kappa=12.5$ (triangles). Lines show the hyperradial density $\left|F_{0 q}(x)\right|^{2}$, Eq. (12). The $s_{0}$ entering into Eq. (12) is taken from the ZR model. $q$ is set to 0 for $\kappa=1$ and 6.7, and calculated according to Eq. (10) with $E$ taken from the SV calculations for the other $\kappa$. Note the different scales of the axis in panels (a) and (b).

As discussed in Ref. [48], we also considered systems consisting of two heavy and two light fermions interacting through $V_{\mathrm{g}}$ with $1 / a_{s}=0$ and various angular momenta. The addition of the light particle to the $(2,1)$ system does not, to within our numerical resolution, lead to the appearance of a new resonance. Thus, adding a light particle leaves the system properties largely unchanged. The next subsection shows that adding a heavy fermion to the $(2,1)$ system does lead to the appearance of a new resonance.

\section{B. Four-body resonances}

Unlike the hyperangular Schrödinger equation for the $(2,1)$ system with ZR interactions, that for the $(3,1)$ system with ZR interactions is not analytically soluble. Thus, we employ the SV approach to solve the full Schrödinger equation for FR interactions, and then ana- 


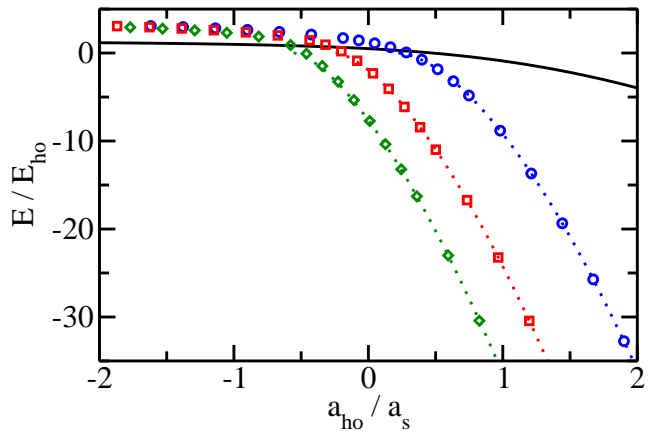

FIG. 14: (Color online) Circles, squares and diamonds show the SV energies for the $(2,1)$ system with $L^{\Pi}=1^{-}$interacting through $V_{\mathrm{g}}$ with $r_{0}=0.005 a_{\mathrm{ho}}$ as a function of $a_{\mathrm{ho}} / a_{s}$ for $\kappa=12,12.314$ and 12.5, respectively. Dotted lines show fits to the SV energies (see text for details). For comparison, the solid line shows the energy for two trapped atoms interacting through $V_{\mathrm{zr}}$.

TABLE I: Extrapolated ZR $s_{0}$ values for small twocomponent Fermi gases at unitarity. The uncertainty of the $s_{0}$ values is estimated to be in the last digit reported. The $(2,2)$ energies for $\kappa=1$ are taken from Ref. 72] and the $(3,2)$ energy for $\kappa=1$ is taken from Ref. [73].

\begin{tabular}{c|cccccc}
\hline \hline$\kappa$ & $s_{0}(3,1)$ & $s_{0}(4,1)$ & $s_{0}(2,2)$ & $s_{0}(2,2)$ & \multirow{2}{*}{$s_{0}(2,2)$} & $s_{0}(3,2)$ \\
& $1^{+}$ & $0^{-}$ & $0^{+}$ & $1^{-}$ & $2^{+}$ & $1^{-}$ \\
\hline 1 & 4.08 & 6.45 & 2.509 & 4.598 & 3.418 & 4.958 \\
2 & 3.86 & 6.15 & 2.575 & 4.357 & & 4.90 \\
4 & 3.51 & 5.68 & 2.754 & 3.997 & 3.478 & 4.85 \\
6 & & & 2.886 & 3.705 & & 4.76 \\
8 & \multirow{2}{*}{2.79} & & 2.947 & 3.430 & 3.326 & \\
10 & & & 2.939 & 3.138 & 3.225 & \\
\hline \hline
\end{tabular}

lyze the eigenenergies and structural properties. In certain cases, we "back out" $s_{0}$ and $q$, i.e., we extract the lowest eigenvalue of the hyperangular Schrödinger equation and the eigenvalues of the radial Schrödinger equation.

The energetically lowest lying state of the $(3,1)$ system has $L^{\Pi}=1^{+}$symmetry for the two-body scattering lengths $a_{s}$ of interest. In the $a_{s} \rightarrow 0^{-}$limit, this can be verified by constructing the wave functions of the noninteracting system. We find numerically that this is also true at unitarity. Figures 15(a) and (b) show the energy of the $(3,1)$ system as a function of $r_{0}$ for different $\kappa$. Similarly to the $(2,1)$ system, the extrapolated ZR energy of the $(3,1)$ system is approached approximately linearly from above for $\kappa=1$. For larger $\kappa$ (i.e., $\kappa=4$ or 8), the ZR limit is approached approximately linearly from below. Table I and squares in Fig. [16] summarize the extrapolated ZR energies for the $(3,1)$ system with
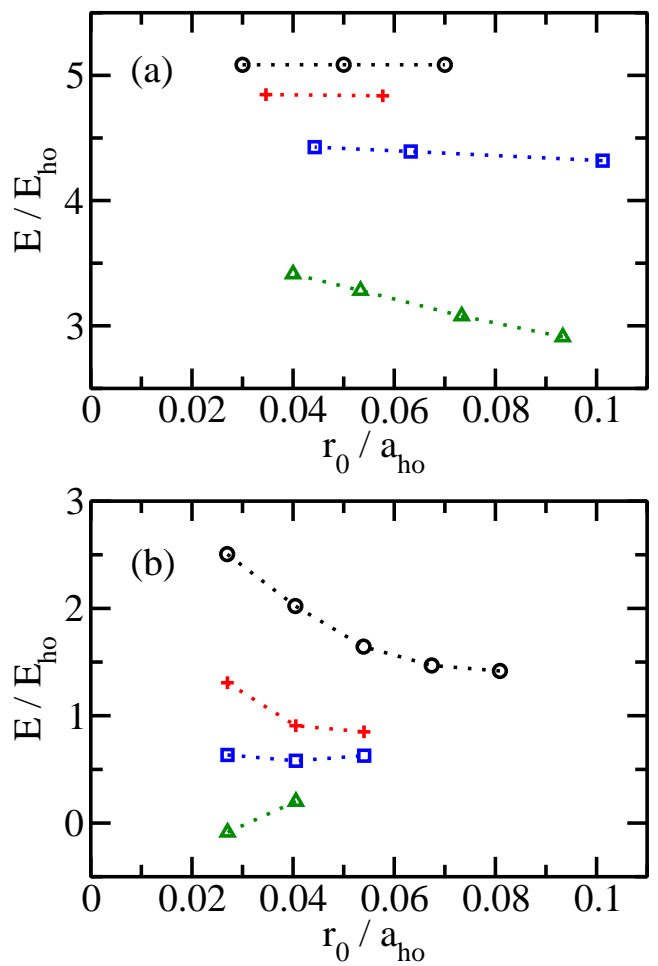

FIG. 15: (Color online) Circles, pluses, squares and triangles show the SV energies for the $(3,1)$ system with $L^{\Pi}=1^{+}$ interacting through $V_{\mathrm{g}}$ at unitarity as a function of $r_{0}$ for (a) $\kappa=1,2,4$ and 8 , and (b) $\kappa=10,10.4,10.5$ and 10.6. Dotted lines are shown as a guide to the eye.

$L^{\Pi}=1^{+}$symmetry for $\kappa \leq 8$. The dependence of the energies on the range $r_{0}$ increases as $\kappa$ increases from 1 or 2 to about 10 , and then becomes comparatively small for $\kappa \approx 10.5$ [see squares in Fig. 15(b)]. For yet larger $\kappa$, the energy varies roughly as $1 / r_{0}^{2}$ and approaches large negative values for small $r_{0}$. In this regime, four-body bound states exist. Although our calculations for larger $\kappa, \kappa>8$, do not allow for a reliable extrapolation of the energies to the $r_{0} \rightarrow 0$ limit, the existence of a four-body or $(3,1)$ resonance around $\kappa \approx 10.4$ or 10.5 is evident.

Although our SV calculations for the $(3,1)$ system are restricted to significantly larger $r_{0}$ than those for the $(2,1)$ system, it is clear that the behavior of the $(3,1)$ energies in the vicinity of the four-body resonance is qualitatively similar to that of the $(2,1)$ energies in the vicinity of the three-body resonance. The main difference is that the four-body resonance occurs at a smaller mass ratio than the three-body resonance. Importantly, the SV energies for the Gaussian two-body potential $V_{\mathrm{g}}$ for the $(3,1)$ system depend - as do those for the $(2,1)$ systemcomparatively weakly on $r_{0}$ in the vicinity of the resonance [squares in Fig. [15(b)]. The qualitatively similar dependence of the energies of the $(3,1)$ and $(2,1)$ sys- 


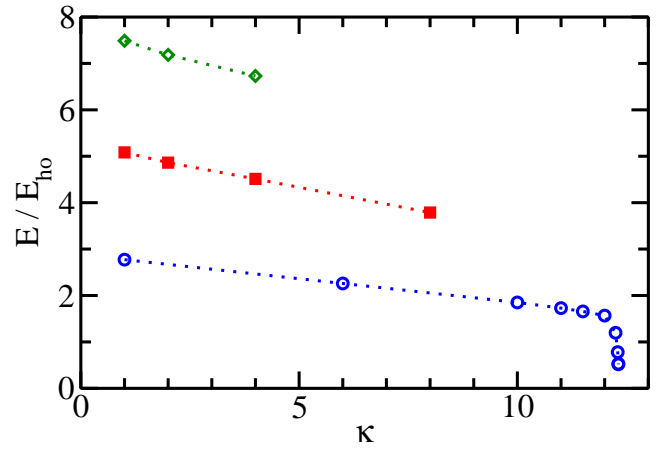

FIG. 16: (Color online) Circles, squares and diamonds show the extapolated ZR energies as a function of $\kappa$ for the $(2,1)$ system with $L^{\Pi}=1^{-}$symmetry, the $(3,1)$ system with $L^{\Pi}=$ $1^{+}$symmetry, and the $(4,1)$ system with $L^{\Pi}=0^{-}$symmetry, respectively. As a guide to the eye, dotted lines connect data points. For the $(3,1)$ and $(4,1)$ systems, our FR SV energies allow for a reliable extrapolation to the $r_{0} \rightarrow 0$ limit only for relatively small $\kappa$.

tems on $r_{0}$ suggests that the four-body resonance occurs when $s_{0}$ is approximately equal to $1 / 2$. More specifically, applying the framework detailed in Sec. IIB i.e., assuming that the hyperradial and the hyperangular motion separate not only for ZR interactions but also for FR interactions, the relatively weak dependence of the $(3,1)$ energies for $\kappa \approx 10.5$ implies that the four-body resonance occurs when $s_{0}$ is approximately $1 / 2$. This interpretation assumes separability or approximate separability of the hyperangular and hyperradial parts of the wave function. If the coupling between different channel functions was appreciable for the FR interactions considered in our SV calculations, then the hyperspherical framework of Sec. IIB would need to be modified. While it is possible that the dependence of our SV energies on $r_{0}$ is consistent with a value of $s_{0}$ notably different from $1 / 2$ and non-vanishing channel coupling, we believe that the former scenario (i.e., approximate separability and $s_{0} \approx 1 / 2$ in the vicinity of the four-body resonance) is more likely. This conclusion is supported by our analysis of the hyperradial densities.

Symbols in Fig. 17 show the hyperradial density $P_{\text {hyper }}(R)$ for the $(3,1)$ system with $\kappa=1-10.6$ interacting through the $\mathrm{FR}$ two-body potential $V_{\mathrm{g}}$ with various $r_{0}$. For $\kappa \leq 8$, we find that the hyperradial densities for the Gaussian potential $V_{\mathrm{g}}$ (symbols) are well reproduced by $\left|F_{0 q}(R)\right|^{2}$, Eq. (12), with $q$ set to zero and $s_{0}$ determined by fitting the SV densities to Eq. (12) [solid lines in Fig. 17(a)]. Alternatively, we set $q=0$ and use the SV energies to determine $s_{0}$ via Eq. (11). For $\kappa=1$ and 4 , the resulting hyperradial densities are indistinguishable from the lines shown Fig. 17)(a). For $\kappa=8$, however, the description that treats $s_{0}$ as a fit-

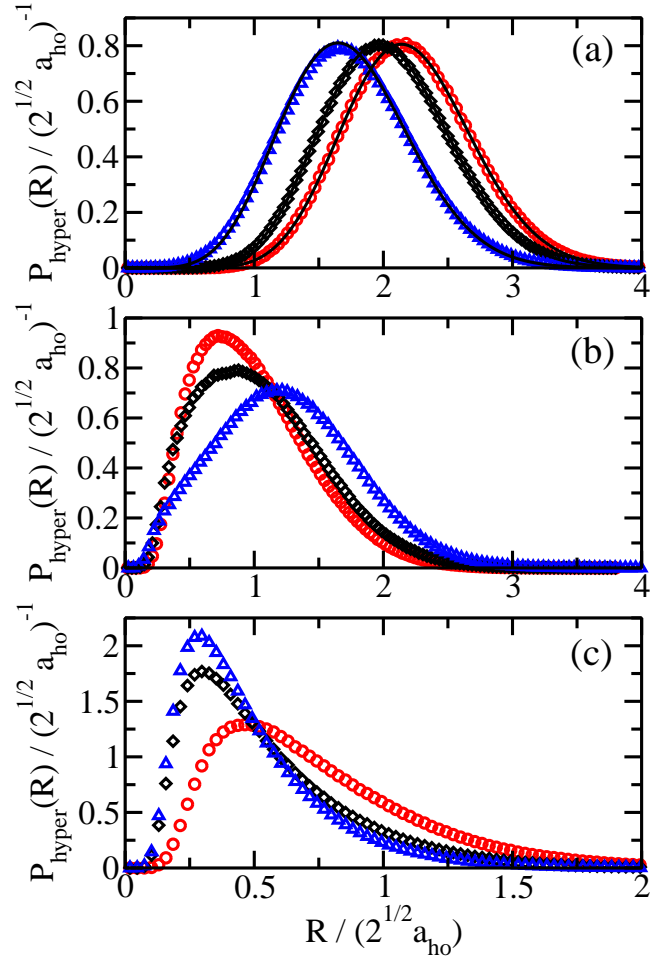

FIG. 17: (Color online) Hyperradial density $P_{\text {hyper }}(R)$ of the $(3,1)$ system with $L^{\Pi}=1^{+}$at unitary. Symbols show the results from the SV calculation where the heavy-light particles interact through $V_{\mathrm{g}}(r)$ for (a) $\kappa=1$ and $r_{0}=0.03 a_{\text {ho }}$ (circles), $\kappa=4$ and $r_{0} \approx 0.044 a_{\text {ho }}$ (diamonds), and $\kappa=8$ and $r_{0}=$ $0.04 a_{\mathrm{ho}}$ (triangles); (b) $\kappa=10$ and $r_{0} \approx 0.054 a_{\mathrm{ho}}$ (circles), $\kappa=10$ and $r_{0} \approx 0.040 a_{\text {ho }}$ (diamonds), and $\kappa=10$ and $r_{0} \approx$ $0.027 a_{\mathrm{ho}}$ (triangles); and (c) $\kappa=10.4$ and $r_{0} \approx 0.041 a_{\mathrm{ho}}$ (circles), $\kappa=10.5$ and $r_{0} \approx 0.027 a_{\text {ho (diamonds), and } \kappa=}$ 10.6 and $r_{0} \approx 0.027 a_{\text {ho }}$ (triangles). In panel (a), lines show the hyperradial density $\left|F_{0 q}(R)\right|^{2}$, Eq. (12), with $q$ set to zero and $s_{0}$ determined by fitting the SV density to Eq. (12). Note the different scales of the axis in panels (a)-(c).

ting parameter leads to a better description. Symbols in Fig. 17(b) show the hyperradial densities for $\kappa=10$ and three different $r_{0}$ values. As $r_{0}$ decreases, the the hyperradial density moves to larger $R$ values and approaches the hyperradial density expected for universal states. Symbols in Fig. 17(c) show the hyperradial densities for $\kappa=10.4,10.5$ and 10.6. As $\kappa$ increases, the maximum of $P_{\text {hyper }}(R)$ increases and moves to smaller $R$ values. Moreover, the tail of $P_{\text {hyper }}(R)$ for $\kappa=10.4-10.6$ starts to resemble that of a bound state. The ZR model, Eq. (12), qualitatively but not quantitatively reproduces the FR hyperradial densities for $\kappa=10-10.6$ if the normalization constant $N_{0 q}, q$ and $s_{0}$ are treated as fitting parameters. Based on a detailed analysis of the hyperradial densities of the $(2,1)$ system for various $r_{0}$, we 
believe that the ZR model would reproduce the FR hyperradial densities of the $(3,1)$ system quantitatively if $r_{0}$ was smaller.

Our calculations suggest that the $s_{0}$ for the $(3,1)$ system with $L^{\Pi}=1^{+}$symmetry changes notably over a small range of mass ratios. In particular, we find $s_{0} \approx 1 / 2$ for $\kappa=10.4$ or 10.5. Recently, Castin and coworkers [76] reported that $s_{0}$ becomes zero for $\kappa=13.384$. While our $s_{0}$ values are not in direct contradiction with this finding, an extrapolation of our results suggests that the $s_{0}$ value of the $(3,1)$ system goes to zero at a smaller mass ratio than that found by Castin and coworkers. Future studies need to investigate this question in more detail.

We also treated the energetically lowest-lying state of the $(4,1)$ system at unitarity, which has $L^{\Pi}=0^{-}$symmetry. For this system, our calculations are restricted to the universal regime. Diamonds in Fig. [16] show the extrapolated ZR energies and Table \ shows the corresponding $s_{0}$ values. We find that the hyperradial densities for the FR interaction potential (not shown) are well reproduced by Eq. (12) with $q=0$ and $s_{0}$ determined through a fit or by the SV energy. For completeness, Table I shows selected $s_{0}$ values for the $(3,1),(2,2)$ and $(3,2)$ systems.

\section{CONCLUSIONS}

We considered small fermionic two-component systems under external spherically symmetric confinement with $1 \leq \kappa \lesssim 13.607$, i.e., in regimes where three-body Efimov physics is absent. Our calculations employed two different interaction potentials between the heavy and light atoms, a FR and a ZR interaction potential, and focused primarily on the unitary regime where the interspecies $s$ wave scattering length $a_{s}$ becomes infinitely large. Like atoms were assumed to be non-interacting. To address questions Q1-Q5 (see Sec. I), we performed SV calculations for few-fermion systems that interact through the FR interaction potential $V_{\mathrm{g}}$, and analyzed a subset of the eigenspectrum and selected structural properties such as the hyperradial density as a function of $r_{0}$. The numerical results were interpreted within a hyperspherical framework that becomes exact in the ZR limit. We defined the generalized energy-dependent scattering length $\mathcal{V}_{s_{\nu}}(E)$, which characterizes the $N$-body system in free space. This generalized scattering length is related to the hypervolume introduced in Ref. 77] to characterize bosonic three-particle systems and can be interpreted as being the result of an effective $N$-body force that acts at small (or vanishing) hyperradii $R$. We used the generalized scattering length to connect the properties of the trapped $N$-body system with those of the free-space system.

We found that the $(2,1)$ and $(3,1)$ systems at unitarity interacting through $V_{\mathrm{g}}$ exhibit three-body and four-body resonances when $\kappa \approx 12.314$ and $\kappa \approx 10.4$, respectively. For the $(2,1)$ system with $L^{\Pi}=1^{-}$symmetry, this mass ratio corresponds to, as has been shown by an indepen- dent calculation [46], an $s_{0}$ value of approximately $1 / 2$. For the $(3,1)$ system with $L^{\Pi}=1^{+}$symmetry, the $s_{0}$ value is not known independently. We argued that the $s_{0}$ value of the $(3,1)$ system is approximately $1 / 2$ for around $\kappa=10.4$. It would be extremely valuable if this could be confirmed by an independent calculation that solves the hyperangular Schrödinger equation directly and does not rely, as our analysis, on backing $s_{0}$ out from the full solution. The three- and four-body resonances discussed here are obtained for the interaction potential $V_{\mathrm{g}}$. While the occurance of $N$-body resonances does depend, in general, on the details of the underlying two-body potential, we believe that the results presented here also apply to other short-range interaction potentials. In particular, we argued that $N$-body resonances occur most likely when the $s_{0}$ value that characterizes the $N$-body problem is approximately $1 / 2$. Experimentally, the value of $s_{0}$ can possibly be tuned by varying the lattice confinement or the intraspecies interactions [44]. While our calculations for the $(4,1)$ system were restricted to $\kappa$ values for which the system behaves universally, i.e., for which $N$-body resonances are absent, recent work by Gandolfi and Carlson [49] found that the $(4,1)$ system in free-space exhibits an $N$-body resonance for $\kappa \approx 9.5-9.8$. So far, $N$-body resonances have not been observed for larger systems and future research needs to address whether or not such resonances exist. Our results for the $(2,1)$ and $(3,1)$ systems show, in agreement with Ref. [49], that the energy of the $N$-body bound state changes more rapidly for the $(3,1)$ system than for the $(2,1)$ system for the same change $\Delta \kappa$ of the mass ratio. If $N$-body resonances exist for larger systems, this trend is expected to continue.

Our calculations show that FR effects become increasingly more important as the mass ratio $\kappa$ increases. The dependence on the range of the underlying two-body potential signals the breakdown of universality and has important implications for theory and experiment. Since FR effects can be appreciable, theoretical treatments of unequal-mass Fermi systems based on ZR interactions may not be, in general, sufficient. Our calculations suggest that an analysis of experimental results for few- or many-fermion systems with sufficiently large mass ratio needs to account for non-universal FR effects. Near an $N$-body resonance, the system's lifetime will be reduced due to the formation of molecules. If a two-component Fermi gas is prepared in a regime where $N$-body bound states exist, collapse is expected to set in. While this is, in many instances, an unwanted phenomenon, careful tuning in the vicinity of an $N$-body resonance might open the possibility to study novel physics. On resonance, the $N$-body clusters have a size comparable to that of $s$-wave dimers. At the $(2,1)$ resonance, e.g., the trimer is large. While bound tetramers and pentamers (and presumably larger clusters) exist, their wave functions have negligible overlap with that of the trimer. This suggests that many-body systems with competing dimer and trimer interactions have finite lifetimes and may thus be prepared experimentally. Similarly, many-body systems with com- 
peting two-body and four-body interactions should be experimentally accessible. The study of these many-body systems is expected to uncover novel physics.

\section{ACKNOWLEDGEMENTS}

Support by the NSF through grant PHY-0855332 and the ARO as well as fruitful discussions with S. Tan are gratefully acknowledged.
[1] B. Ricco and M. Ya. Azbel, Phys. Rev. B 29, 1970 (1984).

[2] L. Kofman, A. Linde, and A. A. Starobinsky, Phys. Rev. Lett. 73, 3195 (1994).

[3] L. Gammaitoni, P. Hänggi, P. Jung, and F. Marchesoni, Rev. Mod. Phys. 70, 223 (1998).

[4] C. Chin, R. Grimm, P. Julienne, and E. Tiesinga, Rev. Mod. Phys. 82, 1225 (2010).

[5] W. C. Stwalley, Phys. Rev. Lett. 37, 1628 (1976).

[6] E. Tiesinga, B. J. Verhaar, and H. T. C. Stoof, Phys. Rev. A 47, 4114 (1993).

[7] S. Inouye, M. R. Andrews, J. Stenger, H.-J. Miesner, D. M. Stamper-Kurn, and W. Ketterle, Nature (London) 392, 151 (1998).

[8] S. L. Cornish, N. R. Claussen, J. L. Roberts, E. A. Cornell, and C. E. Wieman, Phys. Rev. Lett. 85, 1795 (2000).

[9] M. Greiner, C. A. Regal, and D. S. Jin, Nature 426, 537 (2003).

[10] M. W. Zwierlein, C. A. Stan, C. H. Schunck, S. M. F. Raupach, S. Gupta, Z. Hadzibabic, and W. Ketterle, Phys. Rev. Lett. 91, 250401 (2003).

[11] T. Bourdel, J. Cubizolles, L. Khaykovich, K. M. F. Magalhāes, S. J. J. M. F. Kokkelmans, G. V. Shlyapnikov, and C. Salomon, Phys. Rev. Lett. 91, 020402 (2003).

[12] K. E. Strecker, G. B. Partridge, and R. G. Hulet, Phys. Rev. Lett. 91, 080406 (2003).

[13] C. A. Regal, M. Greiner, and D. S. Jin, Phys. Rev. Lett. 92, 040403 (2004).

[14] M. W. Zwierlein, C. A. Stan, C. H. Schunck, S. M. F. Raupach, A. J. Kerman, and W. Ketterle, Phys. Rev. Lett. 92, 120403 (2004).

[15] J. Kinast, S. L. Hemmer, M. E. Gehm, A. Turlapov, and J. E. Thomas, Phys. Rev. Lett. 92, 150402 (2004).

[16] A. Schirotzek, C.-H. Wu, A. Sommer, and M. W. Zwierlein, Phys. Rev. Lett. 102, 230402 (2009).

[17] K. Xu, T. Mukaiyama, J. R. Abo-Shaeer, J. K. Chin, D. E. Miller, and W. Ketterle, Phys. Rev. Lett. 91, 210402 (2003).

[18] S. Jochim, M. Bartenstein, A. Altmeyer, G. Hendl, C. Chin, J. Hecker Denschlag, and R. Grimm, Phys. Rev. Lett. 91, 240402 (2003).

[19] J. Cubizolles, T. Bourdel, S. J. J. M. F. Kokkelmans, G. V. Shlyapnikov, and C. Salomon, Phys. Rev. Lett. 91, 240401 (2003).

[20] S. Dürr, T. Volz, A. Marte, and G. Rempe, Phys. Rev. Lett. 92, 020406 (2004).

[21] S. Ospelkaus, A. Pe'er, K.-K. Ni, J. J. Zirbel, B. Neyenhuis, S. Kotochigova, P. S. Julienne, J. Ye, and D. S. Jin, Nature Phys. 4, 622 (2008).

[22] J. G. Danzl, E. Haller, M. Gustavsson, M. J. Mark, R.
Hart, N. Bouloufa, O. Dulieu, H. Ritsch, H.-C. Nägerl, Science 321, 1062 (2008).

[23] J. Deiglmayr, A. Grochola, M. Repp, K. Mörtlbauer, C. Glück, J. Lange, O. Dulieu, R. Wester, and M. Weidemüller, Phys. Rev. Lett. 101, 133004 (2008).

[24] V. N. Efimov, Yad. Fiz. 12, 1080 (1970) [Sov. J. Nucl. Phys. 12, 589 (1971)].

[25] V. Efimov, Nucl. Phys. A 210, 157 (1973).

[26] E. Braaten and H.-W. Hammer, Phys. Rep. 428, 259 (2006).

[27] T. Kraemer, M. Mark, P. Waldburger, J. G. Danzl, C. Chin, B. Engeser, A. D. Lange, K. Pilch, A. Jaakkola, H.-C. Nägerl, R. Grimm, Nature 440, 315 (2006)

[28] S. Knoop, F. Ferlaino, M. Mark, M. Berninger, H. Schöbel, H.-C. Nägerl, and R. Grimm, Nature Physics 5, 227 (2009).

[29] F. Ferlaino, S. Knoop, M. Berninger, W. Harm, J. P. D'Incao, H.-C. Nägerl, and R. Grimm, Phys. Rev. Lett. 102, 140401 (2009).

[30] M. Zaccanti, B. Deissler, C. D'Errico, M. Fattori, M. Jona-Lasinio, S. Müller, G. Roati, M. Inguscio, and G. Modugno, Nature Phys. 5, 586 (2009).

[31] S. E. Pollack, D. Dries, and R. G. Hulet, Science 326, 1683 (2009).

[32] J. von Stecher, J. P. D'Incao, and C. H. Greene, Nature Physics 5, 417 (2009).

[33] J. P. D'Incao, J. von Stecher, and C. H. Greene, Phys. Rev. Lett. 103, 033004 (2009).

[34] H.-W. Hammer and L. Platter, Eur. Phys. J. A 32, 113 (2007).

[35] D. S. Petrov, Phys. Rev. A 67, 010703(R) (2003).

[36] J. P. D'Incao and B. D. Esry, Phys. Rev. A 73, 030702(R) (2006).

[37] J. P. D'Incao and B. D. Esry, Phys. Rev. A 73, 030703(R) (2006).

[38] T. B. Ottenstein, T. Lompe, M. Kohnen, A. N. Wenz, and S. Jochim, Phys. Rev. Lett. 101, 203202 (2008).

[39] A. N. Wenz, T. Lompe, T. B. Ottenstein, F. Serwane, G. Zürn, and S. Jochim, Phys. Rev. A 80, 040702 (2009).

[40] J. H. Huckans, J. R. Williams, E. L. Hazlett, R. W. Stites, and K. M. O'Hara, Phys. Rev. Lett. 102, 165302 (2009).

[41] J. R. Williams, E. L. Hazlett, J. H. Huckans, R. W. Stites, Y. Zhang, K. M. O'Hara, Phys. Rev. Lett. 103, 130404 (2009).

[42] F. Werner and Y. Castin, Phys. Rev. A 74, 053604 (2006).

[43] J. von Stecher, C. H. Greene, and D. Blume, Phys. Rev. A 76, 053613 (2007).

[44] Y. Nishida, D. T. Son, and S. Tan, Phys. Rev. Lett. 100, 090405 (2008). 
[45] A. Gezerlis, S. Gandolfi, K. E. Schmidt, and J. Carlson, Phys. Rev. Lett. 103, 060403 (2009).

[46] O. I. Kartavtsev and A. V. Malykh, J. Phys. B 40, 1429 (2007).

[47] S. T. Rittenhouse, N. P. Mehta, and C. H. Greene, Phys. Rev. A 82, 022706 (2010).

[48] D. Blume and K. M. Daily, arXiv:1006.5002 accepted for publication in Physical Review Letters.

[49] S. Gandolfi and J. Carlson, arXiv:1006.5186.

[50] For Li-K, see M. Taglieber, A.-C. Voigt, T. Aoki, T. W. Hänsch, and K. Dieckmann, Phys. Rev. Lett. 100, 010401 (2008). A number of groups are currently pursuing the trapping and cooling of mixtures containing combinations of $\mathrm{Li}, \mathrm{K}, \mathrm{Sr}$ and $\mathrm{Yb}$.

[51] R. G. Newton, Scattering Theory of Waves and Particles, 2nd Ed. (Dover Publications, Inc., Mineola, New York).

[52] E. Fermi, Nuovo Cimento 11, 157 (1934).

[53] K. Huang and C. N. Yang, Phy. Rev. 105, 767 (1957).

[54] T. Busch, B.-G. Englert, K. Rzążewski, and M. Wilkens, Foundations of Phys. 28, 549 (1998).

[55] L. M. Delves, Nucl. Phys. 9, 391 (1958).

[56] L. M. Delves, Nucl. Phys. 20, 275 (1960).

[57] J. Macek, J. Phys. B 1, 831 (1968).

[58] C. D. Lin, Phys. Rep. 257, 1 (1995).

[59] D. Blume, J. von Stecher, and C. H. Greene, Phys. Rev. Lett. 99, 233201 (2007).

[60] J. von Stecher, C. H. Greene and D. Blume, Phys. Rev. A 77, 043619 (2008).

[61] F. Werner and Y. Castin, Phys. Rev. Lett. 97, 150401 (2006).

[62] B. Borca, D. Blume, and C. H. Greene, New J. Phys. 5, 111 (2003). This reference treats the trapped two-particle system with $s$-wave ZR interactions. In the notation of the present paper, this corresponds to $s_{\nu}=1 / 2$.

[63] We use $A_{\nu q}=\sqrt{\Gamma\left(q+1+s_{\nu}\right) / \Gamma(q+1)} / \Gamma\left(1+s_{\nu}\right)$ and $B_{\nu q}=-\sqrt{\Gamma(q+1) / \Gamma\left(q+1+s_{\nu}\right)} \cos (\pi q) /[\Gamma(1-$ $\left.\left.s_{\nu}\right) \cos \left(\pi\left(q+s_{\nu}-1 / 2\right)\right)\right]$.
[64] This can be seen by considering the small $x$-behavior of $\left|g_{\nu q}(x)\right|^{2}$ for $s_{\nu}=1,\left|g_{\nu q}(x)\right|^{2} \rightarrow 1 / x$ for $s_{\nu}=1$. Integrating $\left|g_{\nu q}(x)\right|^{2}$ over $x$ from $x=0$ to $x=\epsilon$ gives $\left.\ln x\right|_{0} ^{\epsilon}+C$ (where $C$ is a constant), which diverges. Thus, the wave function for $s_{\nu} \geq 1$ is not normalizable unless $g_{\nu q}(x)$ is eliminated.

[65] If $s_{\nu}+q$ or $q$ are equal to an integer, Eq. (13) contains, as written, infinities. In these cases, $N_{\nu q}$ reduces to $N_{\nu q}=$ $\sqrt{2 /\left[\Gamma(q+1) \Gamma\left(q+1+s_{\nu}\right)\right]}$.

[66] B. J. Verhaar, J. P. H. W. van den Eijnde, M. A. J. Voermans, and M. M. J. Schaffrath, J. Phys. A 17, 595 (1984).

[67] K. Kanjilal and D. Blume, Phys. Rev. A 73, 060701 (2006).

[68] Y. Suzuki and K. Varga, Stochastic Variational Approach to Quantum Mechanical Few-Body Problems (Springer Verlag, Berlin, 1998).

[69] H. H. B. Sørensen, D. V. Fedorov and A. S. Jensen, in Nuclei and Mesoscopic Physics, AIP Conf. Proc. No. 777, edited by V. Zelevinsky (AIP, Melville, NY, 2005), p. 12.

[70] J. von Stecher and C. H. Greene, Phys. Rev. Lett. 99, 090402 (2007).

[71] G. V. Skorniakov and K. A. Ter-Martirosian, Zh. Eksp. Teor. Fiz. 31, 775 (1956) [Sov. Phys. JETP 4, 648 (1957)].

[72] K. M. Daily and D. Blume, Phys. Rev. A 81, 053615 (2010).

[73] D. Blume and K. M. Daily, arXiv:1008.3191.

[74] B. L. Hammond, W. A. Lester, Jr., and P. J. Reynolds, Monte Carlo Methods in Ab Initio Quantum Chemistry (World Scientific, Singapore, 1994).

[75] For equal masses, the labels "heavy" and "light" are replaced by "spin-up" and "spin-down".

[76] Y. Castin, C. Mora, and L. Pricoupenko, arXiv: 1006.4720

[77] S. Tan, Phys. Rev. A 78, 013636 (2008). 